\title{
Hensel-lifting torsion points on Jacobians and Galois representations
}

\author{
Nicolas Mascot* \\ AUB, Beirut, Lebanon
}

April 1, 2019

\begin{abstract}
Let $\rho$ be a mod $\ell$ Galois representation. We show how to compute $\rho$ explicitly, given the characteristic polynomial of the image of the Frobenius at one prime $p$ and a curve $C$ whose Jacobian contains $\rho$ in its $\ell$-torsion. The main ingredient is a method to $p$-adically lift torsion points on a Jacobian in the framework of Makdisi's algorithms.
\end{abstract}

\section{Acknowledgements}

The author thanks Aurel Page for his implementation of the computation of the Howell form of kernels in Pari/GP and his help with the proof of theorem 2.23 and the formulation of the analysis of the performance of algorithm 13, Aurel Page and Bill Allombert for their help with Pari/GP's C language library, and Kamal Khuri-Makdisi for some useful conversations.

The computer algebra packages used for the computations presented in this paper were almost exclusively Pari/GP, plus a little bit of Magma and GAP18].

Keywords: Galois representation, Jacobian, p-adic, algorithm.

\section{Introduction}

\subsection{Statement of the problem and notations}

The notations set in this section will be used throughout the rest of this article.

Let $\ell \in \mathbb{N}$ be a prime number, let $d \in \mathbb{N}$, and let $\rho: \operatorname{Gal}(\overline{\mathbb{Q}} / \mathbb{Q}) \longrightarrow \mathrm{GL}_{d}\left(\mathbb{F}_{\ell}\right)$ be a $\bmod \ell$ Galois representation of degree $d$. We would like to compute $\rho$, that is to say determine explicitly a polynomial $F(x) \in \mathbb{Q}[x]$ whose splitting field is the Galois number field corresponding to the kernel of $\rho$, as well as an explicit indexation of the roots of $F(x)$ (in some large enough field where $F(x)$ splits completely) by the points of $\mathbb{F}_{\ell}{ }^{d} \backslash\{0\}$ such that the Galois action on these roots corresponds to the

2010 Mathematics subject classification: 11F80, 11Y40, 14Q05, 14H40, 14G10, 14G15, 14G20. *nm116@aub.edu.lb 
action of $\rho$ on $\mathbb{F}_{\ell}^{d} \backslash\{0\}$. Indeed, given such data and a prime $p \in \mathbb{N}$ at which $\rho$ is unramified, it is not very difficult to determine the image by $\rho$ of the Frobenius at $p$ up to conjugacy in $\operatorname{Im} \rho$, even for very large $p$, thanks to the technique presented in Dok13.

Suppose that we have an explicit model (for instance, equations) for a proper, non-singular, geometrically connected curve $C$ of genus $g$ defined over $\mathbb{Q}$, such that

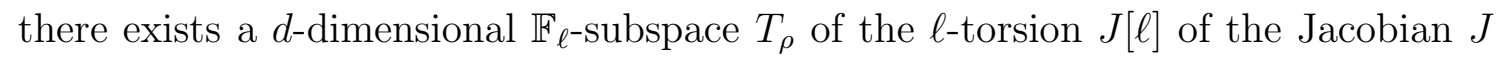
of $C$ such that $T_{\rho}$ affords the Galois representation $\rho$. We wish to use the knowledge of the curve $C$ to compute $\rho$. For example, we showed in Mas13] and Mas18b] how to compute the Galois representations attached to classical modular forms, by taking $C$ to be a modular curve.

In the case when $C$ is a modular curve, we may use the Hecke action in order to single $T_{\rho}$ out of $J[\ell]$ (cf. Mas13] for details), but we have no such tool at our disposal when $C$ is a general curve. Therefore, we instead assume that there is (at least) one prime $p \in \mathbb{N}$ at which $\rho$ is unramified such that we know explicitly the characteristic polynomial $\chi_{\rho}(x) \in \mathbb{F}_{\ell}[x]$ of the image by $\rho$ of the Frobenius $\Phi$ at $p$. We actually make the requirement that $p$ is distinct from $\ell$ and that our model of $C$ has good reduction at $p$ (which implies that $\rho$ is unramified at $p$ by Néron-OggShafarevich). Furthermore, we suppose that the local $L$-factor $L_{p}(x) \in \mathbb{Z}[x]$ of $C$ at $p$ is known explicitly, and that $\chi_{\rho}(x)$, which necessarily divides $L_{p}(x) \bmod \ell$, is actually coprime mod $\ell$ to its cofactor $L_{p}(x) / \chi_{\rho}(x)$. This last requirement ensures that the knowledge of $\chi_{\rho}(x)$ determines the subspace $T_{\rho}$ of $J[\ell]$ uniquely.

The goal of this article is to present an algorithm that, given a model for $C$, the prime $p \in \mathbb{N}$ and the characteristic polynomial $\chi_{\rho}(x)$, computes $\rho$ in the above sense.

Remark 1.1. Since we have an explicit model of $C$, the local $L$-factor $L_{p}(x)$ may in principle be recovered as the numerator of the Hasse-Weil zeta function of the reduction mod $p$ of $C$ by point-counting techniques. Here we assume that we have somehow been able to determine it. In practice, this often means that $p$ cannot be too large.

Remark 1.2. We do not exclude the case where $T_{\rho}=J[\ell]$, i.e. where the representation we want to compute is that afforded by the whole $\ell$-torsion of $J$. In this case, the output of our algorithm may be called an $\ell$-division polynomial of $C$. Thanks to [Dok13], we can then compute the local factor $L_{p^{\prime}}(x) \bmod \ell$ in time polynomial in $\log p^{\prime}$ for primes $p^{\prime} \in \mathbb{N}$ of good reduction. By Chinese remainders, we can thus determine $\# C\left(\mathbb{F}_{p^{\prime n}}\right)$, at least in theory. This approach results in a point-counting method whose complexity is as good as [AGS18, but which is more general since it is not limited to hyperelliptic curves.

Remark 1.3. Although we focus on the case of representations of $\operatorname{Gal}(\overline{\mathbb{Q}} / \mathbb{Q})$ for the simplicity of exposition, it is completely straightforward to generalize the techniques presented in this article to the case of $\bmod \ell$ representations of $\operatorname{Gal}(\bar{k} / k)$ where $k$ is a number field, by taking $C$ to be defined over $k$, and replacing $p$ by a finite prime $\mathfrak{p}$ of $k$ whose residual characteristic is distinct from $\ell$ and at which $C$ has good reduction.

Remark 1.4. The case of a representation with values in $\mathrm{GL}_{d}\left(\mathbb{F}_{\lambda}\right)$, where $\mathbb{F}_{\lambda}$ is a finite extension of $\mathbb{F}_{\ell}$, can also be treated by our methods (provided of course that the curve $C$ is known), since restriction of scalars and the flexibility of [Dok13] allows us to treat it as a representation with values in a subgroup of $\mathrm{GL}_{d\left[\mathbb{F}_{\lambda}: \mathbb{F}_{\ell}\right]}\left(\mathbb{F}_{\ell}\right)$. 


\subsection{Outline}

Our strategy to compute $\rho$ may be summarized as follows:

1. Compute $d$ points forming a basis of the image of $T_{\rho}$ in $J\left(\mathbb{F}_{q}\right)[\ell]$, where $q=p^{a}$ with $a \in \mathbb{N}$ large enough to split $\rho$ (i.e. large enough that the points of the reduction of $T_{\rho} \bmod p$ are defined over $\mathbb{F}_{q}$ ),

2. Fix an integer $e \in \mathbb{N}$, and lift these points to approximations with accuracy $O\left(p^{e}\right)$ of $\ell$-torsion points in $J\left(\mathbb{Q}_{q}\right)$, that is to say to points of $J\left(\mathbb{Z}_{q} / p^{e}\right)$, where $\mathbb{Q}_{q}$ denotes the unramified extension of $\mathbb{Q}_{p}$ whose residue field is $\mathbb{F}_{q}$ and $\mathbb{Z}_{q}$ is the ring of integers of $\mathbb{Q}_{q}$,

3. Compute all the $\mathbb{F}_{\ell^{-}}$-linear combinations of these $d$ points, thus obtaining a model of $T_{\rho}$ over $\mathbb{Z}_{q} / p^{e}$,

4. Evaluate a rational map $\alpha: J \rightarrow \mathbb{A}^{1}$ defined over $\mathbb{Q}$ at these $\ell^{d}$ points, and form the monic polynomial $F(x)$ whose roots are these values,

5. Check that these values are distinct, else take another map from $J$ to $\mathbb{A}^{1}$ and try again,

6. Identify $F(x)$ as a polynomial with coefficients in $\mathbb{Q}$, or, if this fails, start over with a larger value of $e$.

Indeed, for each nonzero $t \in T_{\rho}, \alpha(t)$ lies in the field of definition $\mathbb{Q}(t)$ of $t$, so the splitting field of $F(x)$ is a subfield of the field corresponding to the kernel of $\rho$, and agrees with it with high probability since most elements of a number field are primitive elements. More precisely, a sufficient condition for the Galois action on the roots of $F(x)$ to reflect faithfully that on the points of $T_{\rho}$ is that $\alpha$ be injective on $T_{\rho}$, which is what we check at step 5 .

Remark 1.5. Because of the identification performed at step 6 , the output of this algorithm is not guaranteed to be correct, and should be certified by methods such as [Mas18a].

Throughout this algorithm, we use Kamal Khuri-Makdisi's methods [KM04], [KM07] to compute in $J$. We give a brief presentation of these techniques in section 2, including a recipe to construct rational maps from $J$ to $\mathbb{A}^{1}$ and comments on the implementation over a $p$-adic field.

The core of this article is devoted to the presentation of a technique to lift torsion points from $\mathbb{F}_{q}$ to $\mathbb{Q}_{q}$ (up to some arbitrary $p$-adic accuracy) in Makdisi's framework. After differential calculus preliminaries in section 3 , we show how to lift a point from $J\left(\mathbb{F}_{q}\right)$ to $J\left(\mathbb{Z}_{q} / p^{e}\right)$ for any $e \in \mathbb{N}$ in section 4 , and then how to ensure that the lift of an $\ell$-torsion point over $\mathbb{F}_{q}$ remains $\ell$-torsion over $\mathbb{Z}_{q} / p^{e}$ in section 5 .

Finally, we explain in detail how to use these tools to compute Galois representations in section 6, and we give explicit examples in section 7.

Remark 1.6. As we will comment on in the next sections, in Makdisi's method, points on the Jacobian are represented by matrices of a certain size. The crucial point is that most matrices of this given size do not represent any point on the Jacobian; thus lifting a point from $J\left(\mathbb{F}_{q}\right)$ to $J\left(\mathbb{Z}_{q} / p^{e}\right)$ is a non-trivial process. In [Mas13], 
we used Makdisi's methods over $\mathbb{C}$, and we noticed that the accuracy deteriorated little-by-little after each operation in $J$, in that the matrix crept away from the locus corresponding to actual points on the Jacobian. This is the reason that initially prompted us to look for a method to "fix" representations of points on $J$ in Makdisi's method given with poor accuracy. We then realized that performing this "fix" $p$-adically led to an algorithm to compute Galois representations. The methods presented in this article ought to also be able to "fix" complex approximations of points in Makidisi's method, although we have not actually tried them this way.

\section{Computing in the Jacobian}

\subsection{Review of Makdisi's algorithms}

We start by recalling how Makdisi's algorithms work; indeed we will reuse some of their ideas in this article. None of the material present in this subsection is original work.

Remark 2.1. Makdisi's algorithms are very flexible and can be implemented in many different ways, cf. [KM04 and [Bru13] for more details. The version we give here is the one that is best suited to our purpose. Connoisseurs will recognize the medium model in representation $\mathrm{B}_{0}$.

\subsubsection{Representation of spaces of functions on the curve}

As in the previous section, we consider a nice algebraic curve $C$ of genus $g$. However, the ground field $k$ need not be $\mathbb{Q}$ anymore, but could be any perfect field over which one may perform linear algebra effectively. Given a divisor $D$ on $C$, we will denote the corresponding Riemann-Roch space by

$$
\mathcal{L}(D)=\left\{f \in k(C)^{\times} \mid(f)+D \geqslant 0\right\} \cup\{0\} .
$$

We begin by picking an effective divisor $D_{0}$ defined over $k$ on $C$, of degree $d_{0} \geqslant$ $2 g+1$, and we define

$$
V_{n}=\mathcal{L}\left(n D_{0}\right)
$$

for all $n \in \mathbb{N}$. The space $V_{2}$ will play a particular rôle, so we set

$$
V=V_{2}
$$

for brevity.

Let $n_{Z} \geqslant 5 d_{0}+1$ be an integer. We will assume that $k$ is large enough that we can fulfill the following condition:

There exists an effective divisor $Z$ formed of $n_{Z}$ distinct points $P_{i}$ in $C(k) \backslash \operatorname{supp} D_{0}$.

Remark 2.3. For efficiency reasons, we should take $d_{0}$ as close to $2 g+1$ as possible and $n_{Z}$ as close to $5 d_{0}+1$ as possible (bearing in mind constraints such as those described in remark 2.17 and subsection 2.2.5). We will therefore assume that $d_{0}=$ $O(g)$ and that $n_{Z}=O(g)$ for complexity analyses. 
All the computations performed by Makdisi's algorithms take place in the space $V_{5}$ (and so do the ones presented in the article). We represent an element $v \in V_{5}$ by a column vector in $k^{n_{Z}}$ containing its values $v\left(P_{i}\right)$ at the $n_{Z}$ points $P_{i} \in C(k)$. Since such a function has degree at most $5 d_{0}$, this representation is faithful, and defines an embedding of $V_{5}$ into $k^{n_{Z}}$.

In this representation, addition and multiplication of functions is done point-wise, which will simplify our task in the next sections. Given two column vectors $c, c^{\prime} \in$ $k^{n_{Z}}$, we denote by $c \odot c^{\prime}$ their point-wise product, that is to say the column vector whose $i$-th entry is $c_{i} c_{i}^{\prime}$ for all $i$. Thus if $c$ and $c^{\prime}$ represent functions on $C, c \odot c^{\prime}$ represents their product (provided that all these functions lie in $V_{5}$ ).

We will work with subspaces of $V_{5}$. Such a subspace $S$ will be represented by a matrix

$$
M=\left(\begin{array}{ccc}
s_{1}\left(P_{1}\right) & \cdots & s_{\operatorname{dim} S}\left(P_{1}\right) \\
\vdots & & \vdots \\
s_{1}\left(P_{n_{Z}}\right) & \cdots & s_{\operatorname{dim} S}\left(P_{n_{Z}}\right)
\end{array}\right)
$$

of size $n_{Z} \times \operatorname{dim} S$ whose $i, j$-entry is $s_{j}\left(P_{i}\right)$, where the $s_{j} \in S$ form a $k$-basis of $S$ and the $P_{i}$ are as above. Thus the columns of $M$ represent a basis of $S$ in the above sense. Since $S$ has many $k$-bases, this representation is of course not unique. We will call such a matrix a matrix representing $S$, or a matrix for $S$.

Given a column $c \in k^{n_{Z}}$ and a matrix $M$ with $n_{Z}$ rows, we denote by $c \odot M$ the matrix whose column are the $c \odot M_{j}$, where the $M_{j}$ are the columns of $M$. Thus if $c$ represents a function $f$ and $M$ represents a subspace $S$ of $V_{5}$, then $f \odot M$ represents the subspace $f S=\{f s, s \in S\}$ (provided that this is still a subspace of $V_{5}$ ).

Later on, we will frequently need to compute a matrix $K_{S}$ of size $\left(n_{Z}-\operatorname{dim} S\right) \times n_{Z}$ whose rows represent independent linear equations defining $S$ as a subspace of $k^{n_{Z}}$. Such a matrix can be obtained simply as the transpose of the kernel of the transpose of $M$, and will be referred to as an equation matrix for $S$.

Conversely, given a matrix $A$ whose rows represent linear equations, we call a matrix whose columns represent a basis of the space of solutions of these linear equations a kernel matrix of $A$.

Remark 2.4. In this article, we will actually work with $k=\mathbb{F}_{q}$ or $\mathbb{Q}_{q}$, where $q=p^{a}$ is a prime power, and we impose that the $n_{Z}$ points $P_{i} \in C(k)$ remain distinct $\bmod p$. The Hasse bounds show that this is always achievable, possibly at the price of enlarging $a$, and also that $q$ cannot be very small. We will also suppose that matrices representing a subspace $S$ of $V_{5}$, as well as kernel (resp. equation) matrices, have coefficients in the ring of integers $\mathbb{Z}_{q}$ of $\mathbb{Q}_{q}$, and that their columns (resp. rows) remain linearly independent when reduced $\bmod p$. We will show how to preserve this condition throughout our computations, and more generally how to perform linear algebra over $\mathbb{Z}_{q}$ without loss of $p$-adic accuracy, in section 2.2.4 below.

By Riemann-Roch, every point $x \in J=\operatorname{Pic}^{0}(C)$ is of the form $x=\left[D-D_{0}\right]$ for some (non-unique) effective divisor $D$ of degree $d_{0}$. Such a point will be represented by a matrix $W_{D}$ for the $d_{0}$-codimensional subspace $\mathcal{L}\left(2 D_{0}-D\right) \subsetneq V$ of $V$. Thus $x$ is represented by an effective divisor $D$ such that $\left[D-D_{0}\right]=x$, itself represented by the subspace $\mathcal{L}\left(2 D_{0}-D\right)$, itself represented by a matrix $W_{D}$ of size $n_{Z} \times d_{W}$, where

$$
d_{W}=d_{0}+1-g
$$


is the dimension of such a subspace. Since $\operatorname{deg}\left(2 D_{0}-D\right)=d_{0} \geqslant 2 g+1$, the RiemannRoch space $\mathcal{L}\left(2 D_{0}-D\right)$ is base-point-free, so this representation is faithful. However, it is obviously not unique.

In particular, the space $V_{1}=\mathcal{L}\left(D_{0}\right)$ represents the origin of $J$, so we denote by $W_{0}$ a matrix representing this space (thus $W_{0}$ means $W_{D}$ for $D=D_{0}$, not $D=0$ ).

We suppose that we can compute an explicit basis of $V_{1}=\mathcal{L}\left(D_{0}\right)=W_{0}$. This presents various difficulties depending on what kind of model of $C$ we have, cf. for instance [Hes02]. Thanks to algorithm 1 introduced below, we can then compute matrices representing $V_{n}$ for any $n \in \mathbb{N}$. We initialize Makdisi's algorithms by precomputing matrices $W_{0}, M_{V}$, and $M_{V_{3}}$ representing respectively $V_{1}, V=V_{2}$, and $V_{3}$, as well as equation matrices $K_{V}$ and $K_{V_{3}}$ for $V$ and $V_{3}$. Although all the action will take place in $V_{5}$, we do not need to compute a matrix (nor an equation matrix) for $V_{5}$.

Remark 2.5. The dissension between the notations $M_{V}, M_{V_{3}}$ (matrices for $\mathcal{L}\left(2 D_{0}\right)$ and $\left.\mathcal{L}\left(3 D_{0}\right)\right)$ and $W_{D}$ (matrix for the subspace $\mathcal{L}\left(2 D_{0}-D\right)$ representing $\left[D-D_{0}\right] \in$ $J$ ) is meant as a reminder that $M_{V}$ and $M_{V_{3}}$ are "structure constants" describing the curve $C$, whereas $W_{D}$ represents a (variable) point on $J$.

\subsubsection{Arithmetic on divisors}

Before we present Makdisi's algorithms per se, we show how we can add and subtract divisors at the level of Riemann-Roch spaces. Let us start with addition. We set the following notation: given two subspaces $S, T \subset k^{n_{Z}}$, we let $S \cdot T$ be the space spanned by the products st where $s \in S$ and $t \in T$.

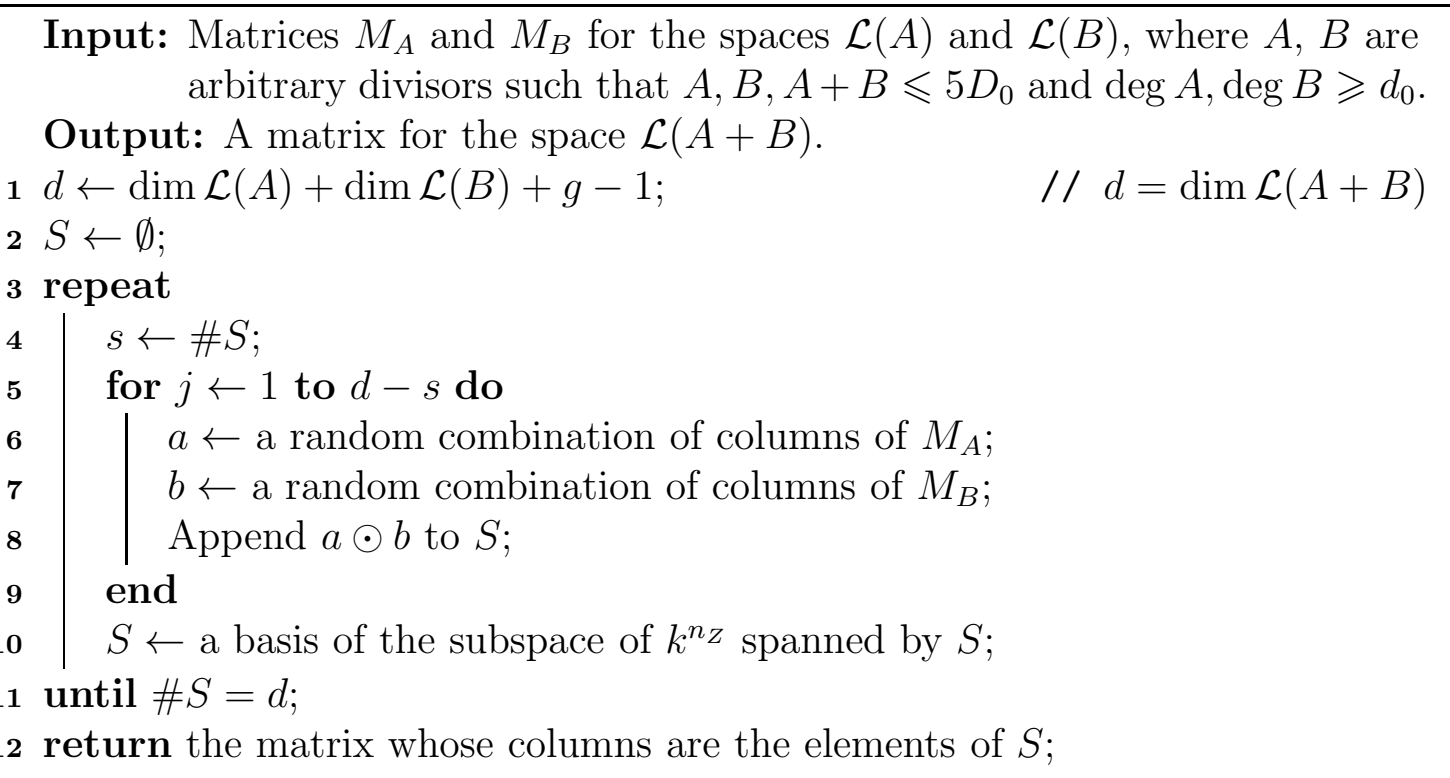

\section{Algorithm 1: DivAdd}

Proof. Makdisi proves in [KM04, lemma 2.2] that if $A$ and $B$ both have degree at least $2 g+1$, then the multiplication map

$$
\mathcal{L}(A) \otimes \mathcal{L}(B) \longrightarrow \mathcal{L}(A+B)
$$

is surjective; in other words, we have $\mathcal{L}(A+B)=\mathcal{L}(A) \cdot \mathcal{L}(B)$. The dimension of this space is computed at line 1 by Riemann-Roch. In each iteration of the for 
loop, $a$ (resp. $b$ ) represents a random element of $\mathcal{L}(A)$ (resp. $\mathcal{L}(B))$, so $a \odot b$ represents an element of $\mathcal{L}(A) \cdot \mathcal{L}(B)=\mathcal{L}(A+B)$. We keep forming such products until we generate $\mathcal{L}(A+B)$.

The condition $A, B, A+B \leqslant 5 D_{0}$ ensures that these spaces are subspaces of $V_{5}$, so that their elements are represented faithfully by their values at the points $P_{i}$.

Remark 2.6. If $k$ is a very small finite field, it can be advantageous to execute the for loop at line 5 a little more than $d-\# S$ times so as to ensure we get a generating set of $\mathcal{L}(A+B)$ with high probability (A detailed analysis of the situation is given in [Bru13]). However, our assumption (2.2) implies that $k$ cannot be too small, so that in practice it is extremely rare that we need to execute the repeat...until loop more than once even if we take just $d$ products $a \odot b$.

Remark 2.7. If $k$ is a $p$-adic field and if we follow the conventions set in remark 2.4, the linear algebra for the extraction of a basis of the span of $S$ at line 10 can be performed $\bmod p$ to save time.

We now show how to subtract divisors at the level of the Riemann-Roch spaces. We begin with the case where the dimension of the result is known in advance, which is frequently the case thanks to the Riemann-Roch theorem.

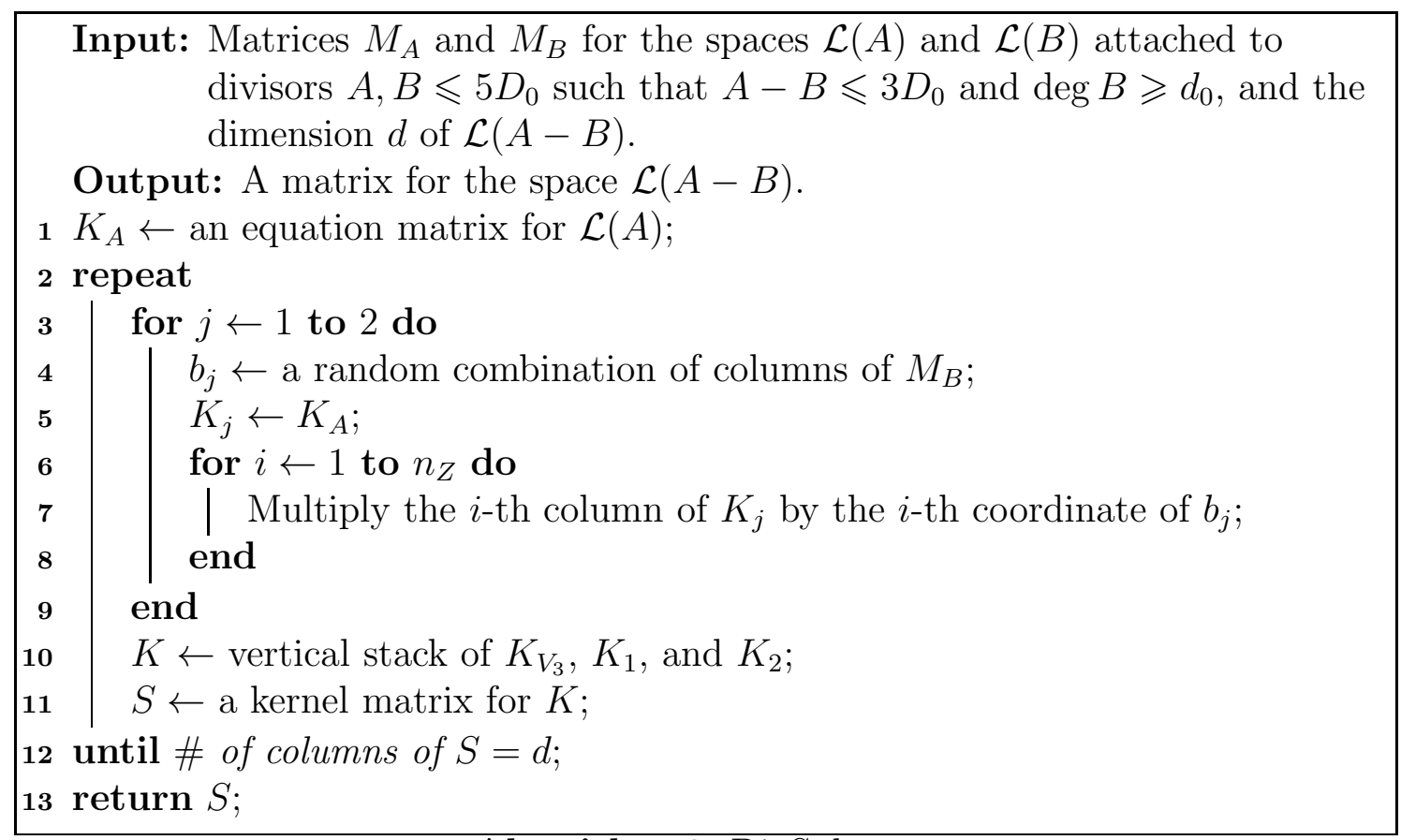

Algorithm 2: DivSub

Proof. As in algorithm 1, the condition $A, B, A-B \leqslant 5 D_{0}$ ensure that the elements of the corresponding Riemann-Roch spaces are faithfully represented by their evaluation at $Z$.

Since $\operatorname{deg} B \geqslant d_{0}>2 g$, the space $\mathcal{L}(B)$ is base-point-free; in other words, the inequality

$$
\inf _{f \in \mathcal{L}(B)}(f) \geqslant-B
$$

is actually an equality. It follows that

$$
\mathcal{L}(A-B)=\left\{v \in V_{5} \mid v \mathcal{L}(B) \subset \mathcal{L}(A)\right\}
$$


Actually, we have $\mathcal{L}(A-B)=\left\{v \in V_{3} \mid v \mathcal{L}(B) \subset \mathcal{L}(A)\right\}$ since $A-B \leqslant 3 D_{0}$.

Now, $b_{1}$ and $b_{2}$ represent random elements $f_{1}$ and $f_{2}$ of $\mathcal{L}(B)$, and the matrix $S$ computed at line 11 represents $\operatorname{ker} K_{V_{3}} \cap \operatorname{ker} K_{1} \cap \operatorname{ker} K_{2}$. But the first of these kernels represents precisely $V_{3}$ by definition of $K_{V_{3}}$, whereas the last two represent $\left\{v \in k^{n_{Z}} \mid v \odot b_{j} \in \mathcal{L}(A)\right\}$ for $j=1,2$. Thus $S$ represents the subspace $L=\left\{v \in V_{3} \mid v f_{1}, v f_{2} \in \mathcal{L}(A)\right\}$ of $V_{5}$.

If the inequality

$$
\inf \left(\left(f_{1}\right),\left(f_{2}\right)\right) \geqslant-B
$$

is actually an equality, then $L$ is exactly $\mathcal{L}(A-B)$ by the same logic as above. Else, $L$ is a strict super-space of $\mathcal{L}(A-B)$, but we can detect this by comparing its dimension to the known dimension of $\mathcal{L}(A-B)$, so we simply try again with another choice of $b_{1}$ and $b_{2}$.

Remark 2.8. As pointed out in section 3 of [KM07], the condition inf $\left(\left(f_{1}\right),\left(f_{2}\right)\right)=$ $-B$ can be understood as the condition that two elements of an ideal of a Dedekind domain generate that ideal. This explains why we must take at least two elements $f_{1}, f_{2} \in \mathcal{L}(B)$. This condition will then be satisfied with high probability, except if $k$ is a very small finite field, in which case it can be a good idea to consider more than two elements $f_{1}, f_{2} \in \mathcal{L}(B)$ (cf. Bru13 for a detailed analysis). Due to assumption (2.2), $k$ cannot be too small, so two elements are enough in practice.

In the case where $\operatorname{dim} \mathcal{L}(A-B)$ is not known in advance, we can still compute $\mathcal{L}(A-B)$ by taking $b_{j}$ ranging over the columns $M_{B}$, which represent a basis $\left(f_{j}\right)$ of $\mathcal{L}(B)$ so that $\inf \left(f_{j}\right)=-B$. Of course, this leads to solving a larger linear system, and is therefore slower, than with two random elements $f_{1}, f_{2} \in \mathcal{L}(B)$.

This more difficult case is in particular needed in the following situation: given matrices $W_{D}, W_{D^{\prime}}$ representing points $x=\left[D-D_{0}\right], x^{\prime}=\left[D^{\prime}-D_{0}\right] \in J$, we can test whether $x=x^{\prime}$, that is to say whether $D \sim D^{\prime}$, since $\operatorname{DivSub}\left(W_{D}, W_{D^{\prime}}\right)$ represents $\mathcal{L}\left(D-D^{\prime}\right)$ which has dimension 1 in this case, and 0 else. In particular, we can test whether $W_{D}$ represents $0 \in J$ by taking $W_{D^{\prime}}=W_{0}$.

\subsubsection{Arithmetic in the Jacobian}

Thanks to these two basic operations, Makdisi manages to compute in the Jacobian using only linear algebra operations over $k$. For instance, the following algorithm computes the negative of the sum of two points of $J$. Since we have the representation $W_{0}$ of $0 \in J$, we can then perform additions and subtractions in $J$.

Input: Matrices $W_{1}$ and $W_{2}$ for the spaces $\mathcal{L}\left(2 D_{0}-D_{1}\right)$ and $\mathcal{L}\left(2 D_{0}-D_{2}\right)$ encoding the points $x_{1}=\left[D_{1}-D_{0}\right]$ and $x_{2}=\left[D_{2}-D_{0}\right]$ of $J$.

Output: A matrix for a space $\mathcal{L}\left(2 D_{0}-D_{3}\right)$ encoding $x_{3}=\left[D_{3}-D_{0}\right] \in J$ such that $x_{1}+x_{2}+x_{3}=0$.

$1 W_{12} \leftarrow \operatorname{DivAdd}\left(W_{1}, W_{2}\right)$

$2 W_{12}^{\prime} \leftarrow \operatorname{DivSub}\left(W_{12}, W_{0}\right)$;

$3 c \leftarrow$ a non-zero combination of columns of $W_{12}^{\prime}$;

$/ / \mathcal{L}\left(4 D_{0}-D_{1}-D_{2}\right)$

$/ / \mathcal{L}\left(3 D_{0}-D_{1}-D_{2}\right)$

$/ /$ where $(f)=-3 D_{0}+D_{1}+D_{2}+D_{3}$ for some $D_{3} \geqslant 0$ of degree $d_{0}$

$4 W_{123} \leftarrow c \odot M_{V}$;

$5 W_{3} \leftarrow \operatorname{DivSub}\left(W_{123}, W_{12}^{\prime}\right)$

$/ / \mathcal{L}\left(5 D_{0}-D_{1}-D_{2}-D_{3}\right)$

6 return $W_{3}$;

$/ / \mathcal{L}\left(2 D_{0}-D_{3}\right)$

Algorithm 3: Add-flip 
Note that on both times that we call DivSub, the dimension of the result, namely $d_{W}$, is known in advance by Riemann-Roch.

Finally, although each point of $J$ is represented by a subspace of $V$, itself viewed as a subspace of $k^{n_{Z}}$, clearly not every $d_{W}$-dimensional subspace of $k^{n_{Z}}$ corresponds to a point of $J$. In [KM04], Makdisi gives the following algorithm:

Input: A matrix $W$ of size $n_{Z} \times d_{W}$ with linearly independent columns representing a subspace of $V$.

Output: True if $W$ represents a subspace of the form $\mathcal{L}\left(2 D_{0}-D\right)$ for some effective $D$ of degree $d_{0}$, False else.

$1 w \leftarrow$ a non-zero combination of columns of $W$;

$2 W^{\prime} \leftarrow \operatorname{DivSub}\left(w \odot M_{V}, W\right)$

$3 n \leftarrow$ number of columns of $W^{\prime}$;

4 return True if $n=d_{W}$, False if $n<d_{W}$;

Algorithm 4: Membership test

Proof. The idea is to check whether the elements of the subspace $S$ of $V$ represented by $W$ have many common zeros. Thus the column vector $w$ represents a non-zero element $f$ of this subspace, whose divisor is of the form $(f)=-2 D_{0}+D+D^{\prime}$ where $D$ is the largest divisor such that $W \subset \mathcal{L}\left(2 D_{0}-D\right)$ and $D^{\prime}$ is another effective divisor, so that $W^{\prime}$ represents $\mathcal{L}\left(2 D_{0}-D^{\prime}\right)$. The larger the locus $D$ of common zeros of $S$, the smaller $D^{\prime}$, so the larger $\operatorname{dim} W^{\prime}$. See [KM04, theorem/algorithm 3.14] for more details.

Loosely speaking, the goal of sections 3 and 4 will be to determine the differential of the map $W \mapsto W^{\prime}$, so as to be able to deform the representation of a $p$-adic point of $J$ by a matrix with entries $\bmod p^{e}$ into a representation by a matrix $\bmod p^{2 e}$.

Remark 2.9. Since Makdisi's algorithms involve linear algebra in size $O(g) \times O(g)$, their complexities are $O\left(g^{\omega}\right)$ operations in the ground field, where $2 \leqslant \omega<3$ is such that two matrices of size $n \times n$ can be multiplied in $O\left(n^{\omega}\right)$ operations.

\section{$2.2 \quad$ Extra operations}

We now present complements of ours to Makdisi's algorithms, some of which are inspired by Bru13.

\subsubsection{Fast exponentiation and add-flip chains}

Algorithm 3 does not compute the sum of two points of $J$, but rather their "addflip", that is to say the negative of that sum. Therefore the usual notion of addition chain for fast exponentiation needs to be adapted.

Definition 2.10. Let $m \in \mathbb{Z}$. An add-flip chain for $m$ of length $n \in \mathbb{N}$ is a finite sequence of triples of integers

$$
\left(m_{0}, i_{0}, j_{0}\right),\left(m_{1}, i_{1}, j_{1}\right), \cdots,\left(m_{n}, i_{n}, j_{n}\right)
$$

such that $m_{0}=0, m_{1}=1, m_{n}=m$, and for all $2 \leqslant s \leqslant n$, we have $0 \leqslant i_{s}, j_{s}<s$ and $m_{s}=-m_{i_{s}}-m_{j_{s}}$. 
The value of $i_{0}, j_{0}, i_{1}, j_{1}$ does not matter and is left undefined.

Since we have the representation $W_{0}$ of $0 \in J$, we can perform any addition by performing two consecutive add-flips. Therefore, for all $m \in \mathbb{Z}$ distinct form 0 and 1 , their exists an add-flip chain of length $O(\log |m|)$; and conversely an add-flip chain for $m$ must clearly have length at least $O(\log |m|)$.

Short add-flip chains for a given $m \in \mathbb{Z}$ can be found by putting $m$ in nonadjacent form in the sense of [CF+05, section 9.1.4]. This method has the advantage of being extremely fast. Adapting the continued fraction method described in [BBB94] results in a much slower algorithm that yields chains which are on average only a few percent shorter.

\subsubsection{Pairings}

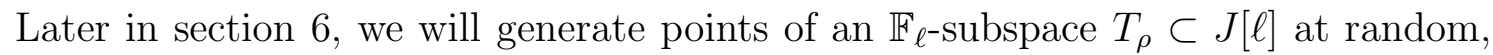
and we will need a tool to detect linear relations between them so as to know whether we have obtained a generating set of $T_{\rho}$ yet. For this, we will use parings.

Let $m \in \mathbb{N}$. The Weil pairing is defined as

$$
\begin{aligned}
e_{m}: \bigwedge^{2} J(k)[m] & \longrightarrow \mu_{m}(k) \\
(x, y) & \longmapsto \frac{f_{x}\left(D_{y}\right)}{f_{y}\left(D_{x}\right)}
\end{aligned}
$$

where $D_{x} \sim x$ and $D_{y} \sim y$ are divisors whose supports do not intersect, and where $f_{x}$ and $f_{y}$ are functions of $C$ such that $\left(f_{x}\right)=m D_{x}$ and $\left(f_{y}\right)=m D_{y}$. It has the disadvantage of being skew-symmetric, which means it is useless for our purpose when the space $T_{\rho}$ is totally isotropic for example. Therefore, we prefer to use the less-known Frey-Rück pairing [FR94]

$$
\begin{aligned}
\{\cdot, \cdot\}_{m}: J(k)[m] \times J(k) / m J(k) & \longrightarrow k^{\times} / k^{\times m} \\
(x, y) & \longmapsto f_{x}\left(D_{y}\right) .
\end{aligned}
$$

In the special case where $k=\mathbb{F}_{q}$ is a finite field containing $\mu_{m}$, we can linearize it by composing it with

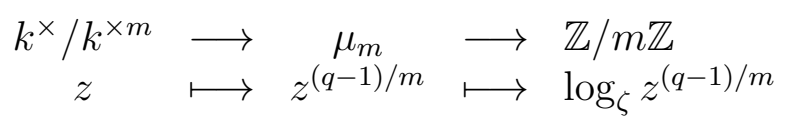

where $\zeta \in \mu_{m}$ is a primitive $m$-th root of 1 and $\log _{\zeta}$ denotes the corresponding discrete logarithm. We will denote the corresponding version of the pairing by

$$
[\cdot, \cdot]_{m}: J\left(\mathbb{F}_{q}\right)[m] \times J\left(\mathbb{F}_{q}\right) / m J\left(\mathbb{F}_{q}\right) \longrightarrow \mathbb{Z} / m \mathbb{Z},
$$

the choice of $\zeta$ being implicit. This pairing is perfect, and allows us to construct linear forms on $J[m]$ simply as $[\cdot, y]_{m}$ for any $y \in J\left(\mathbb{F}_{q}\right)$, and thus to detect linear relations satisfied by a finite collection of points of $J[\mathrm{~m}]$ if we can generate sufficiently many elements $y \in J\left(\mathbb{F}_{q}\right) / m J\left(\mathbb{F}_{q}\right)$.

An implementation of the Frey-Rück pairing in Makdisi's framework is described in Bru13. We describe here our own implementation, which is simpler (for instance it stays in $V_{5}=\mathcal{L}\left(5 D_{0}\right)$ whereas [Bru13] goes up to $\left.\mathcal{L}\left(7 D_{0}\right)\right)$. 
We start with the following auxiliary procedure, which computes the value of a function at a divisor, and may thus be viewed as a generalization in Makdisi's framework of the concept of resultant:

Input: A column $c$ representing a non-zero function $f \in V_{3}$ and a matrix $W_{D}$ representing $\mathcal{L}\left(2 D_{0}-D\right)$, with $\operatorname{deg} D=d_{0}$.

Output: $f(D)$ if the supports of $D$ and $D_{0}$ do not intersect, else FAIL.

$1 w_{1}, \cdots, w_{d_{W}} \leftarrow$ the columns of $W_{D}$, where $d_{W}=\operatorname{dim} V-d_{0}$ as before;

// Find supplement of $\mathcal{L}\left(2 D_{0}-D\right)$ in $V=\mathcal{L}\left(2 D_{0}\right)$

2 Choose columns $s_{1}, \cdots, s_{d_{0}}$ of $M_{V}$ such that $s_{1}, \cdots, s_{d_{0}}, w_{1}, \cdots, w_{d_{W}}$ are linearly independent;

$3 d_{W}^{\prime} \leftarrow 4 d_{0}+1-g ; \quad$ // dimension of $\mathcal{L}\left(5 D_{0}-D\right)$

$4 w_{1}^{\prime}, \cdots, w_{d_{W}^{\prime}}^{\prime} \leftarrow$ columns of $\operatorname{DivAdd}\left(W_{D}, M_{V_{3}}\right) ; \quad$ // $\mathcal{L}\left(5 D_{0}-D\right)$

$/ /$ Find supplement of $\mathcal{L}\left(5 D_{0}-D\right)$ in $V_{5}=\mathcal{L}\left(5 D_{0}\right)$

5 Choose columns $v_{1}, \cdots, v_{d_{0}}$ of $M_{V}$ and $u_{1}, \cdots, u_{d_{0}}$ of $M_{V_{3}}$ such that

$t_{1}, \cdots, t_{d_{0}}, w_{1}^{\prime}, \cdots, w_{d_{W}^{\prime}}^{\prime}$ are linearly independent, where $t_{i} \leftarrow u_{i} \odot v_{i}$

for all $i$;

// Find relations

$6 A \leftarrow$ matrix with columns $s_{1}, \cdots, s_{d_{0}}, t_{1}, \cdots, t_{d_{0}}, w_{1}^{\prime}, \cdots, w_{d_{W}^{\prime}}^{\prime}$;

$7 K \leftarrow$ matrix whose columns form a basis of ker $A$;

$8 K_{s} \leftarrow$ rows 1 to $d_{0}$ of $K$

$9 K_{t} \leftarrow$ rows $d_{0}+1$ to $2 d_{0}$ of $K$

$10 M_{1} \leftarrow K_{t} K_{s}^{-1}$

$11 \Delta_{1} \leftarrow \operatorname{det} M_{1}$

12 if $\Delta_{1}=0$ then FAIL;

// Find relations

$13 A \leftarrow$ matrix with columns $c \odot s_{1}, \cdots, c \odot s_{d_{0}}, t_{1}, \cdots, t_{d_{0}}, w_{1}^{\prime}, \cdots, w_{d_{W}^{\prime}}^{\prime}$;

$14 K \leftarrow$ matrix whose columns form a basis of $\operatorname{ker} A$;

$15 K_{s} \leftarrow$ rows 1 to $d_{0}$ of $K$

$16 K_{t} \leftarrow$ rows $d_{0}+1$ to $2 d_{0}$ of $K$;

$17 M_{f} \leftarrow K_{t} K_{s}^{-1}$

$18 \Delta_{f} \leftarrow \operatorname{det} M_{f}$

19 return $\Delta_{f} / \Delta_{1}$

Algorithm 5: Evaluating a function at a divisor

Proof. View $V_{5}=\mathcal{L}\left(5 D_{0}\right)$ and all its subspaces as embedded into $k^{n_{Z}}$ as explained in section 2, and define

$$
L_{D}=\mathcal{L}\left(2 D_{0}-D\right), \quad L_{D}^{\prime}=\mathcal{L}\left(5 D_{0}-D\right)
$$

for brevity.

We have $V=\mathcal{L}\left(2 D_{0}\right)=S \oplus L_{D}$, where $S$ is the subspace spanned by the $s_{i}$; similarly $V_{5}=\mathcal{L}\left(5 D_{0}\right)=T \oplus L_{D}^{\prime}$ where $T$ is the subspace spanned by the $t_{i}=u_{i} \odot v_{i}$.

By definition,

$$
L_{D}=\left\{v \in V \mid v_{\mid D}=0\right\},
$$

so that $\mathcal{O}_{D} \simeq V / L_{D} \simeq S$; and similarly $\mathcal{O}_{D} \simeq V_{5} / L_{D}^{\prime} \simeq T$.

Since $f \in V_{3}=\mathcal{L}\left(3 D_{0}\right)$, multiplication by $f$ induces a map

$$
\mu_{f}: S \longleftrightarrow V \stackrel{f}{\longrightarrow} V_{5} \longrightarrow T
$$


and $f(D)$ is the determinant of this map seen through the isomorphisms $S \simeq \mathcal{O}_{D}$ and $T \simeq \mathcal{O}_{D}$.

Unfortunately these isomorphisms are not explicit, so it would make no sense to simply take the determinant of the matrix expressing this map on the $s_{i}$ and the $t_{i}$. This is why we divide this determinant by that of the "identity" map

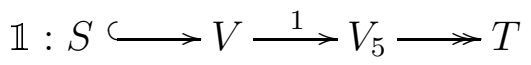

induced by the inclusion of $V$ into $V_{5}$.

More specifically, at line 7 we find relations between the $s_{i}$ and the basis $t_{1}, \cdots, t_{d_{0}}, w_{1}^{\prime}, \cdots, w_{d_{W}^{\prime}}^{\prime}$ of $V_{5}=T \oplus W_{D}^{\prime}$, and we project these relations in $T=$ $V_{5} / W_{D}^{\prime}$ at the next two lines by dropping the rows of $K$ corresponding to the $w_{i}^{\prime}$. The matrix $K_{s}$ formed of the $s$-rows of $K$ is necessarily invertible since $t_{1}, \cdots, t_{d_{0}}, w_{1}^{\prime}, \cdots, w_{d_{W}^{\prime}}^{\prime}$ are linearly independent, so $M_{1}$ is well-defined and is the negative of the matrix of the map $\mathbb{1}$ with respect to the bases $s_{i}$ and $t_{i}$. Its determinant $\Delta_{1}$ is zero iff. there exists a nonzero element of $S$ that lies in $L_{D}^{\prime}$, which means that the supports of $D$ and $D_{0}$ are not disjoint, in which case we return FAIL. Else, by repeating the previous steps with the $c \odot s_{i}$ instead of the $s_{i}$, we get $M_{f}$, the negative of the matrix of $\mu_{f}$, and we recover $f(D)$ as its determinant $\Delta_{f}$ divided by $\Delta_{1}$.

The complexity of this algorithm is $O\left(g^{\omega}\right)$.

Remark 2.11. It is actually more efficient to compute $\Delta_{1}$ as $\operatorname{det}\left(K_{t}\right) / \operatorname{det}\left(K_{s}\right)$ than as $\operatorname{det}\left(K_{t} K_{s}^{-1}\right)$; we show the less efficient way here only for clarity. The same goes of course for $\Delta_{f}$.

Algorithm 5 is only valid for $f \in V_{3}$, which is in general not the case for the function $f_{x}$ in the definition of the Frey-Rück pairing. Therefore the implementation of the pairing is still not completely straightforward. Our solution, inspired by [Bru13], is presented below; we assume that algorithm 3 has been modified so as to return the column $c$ chosen at line 3 as well as $W_{3}$.

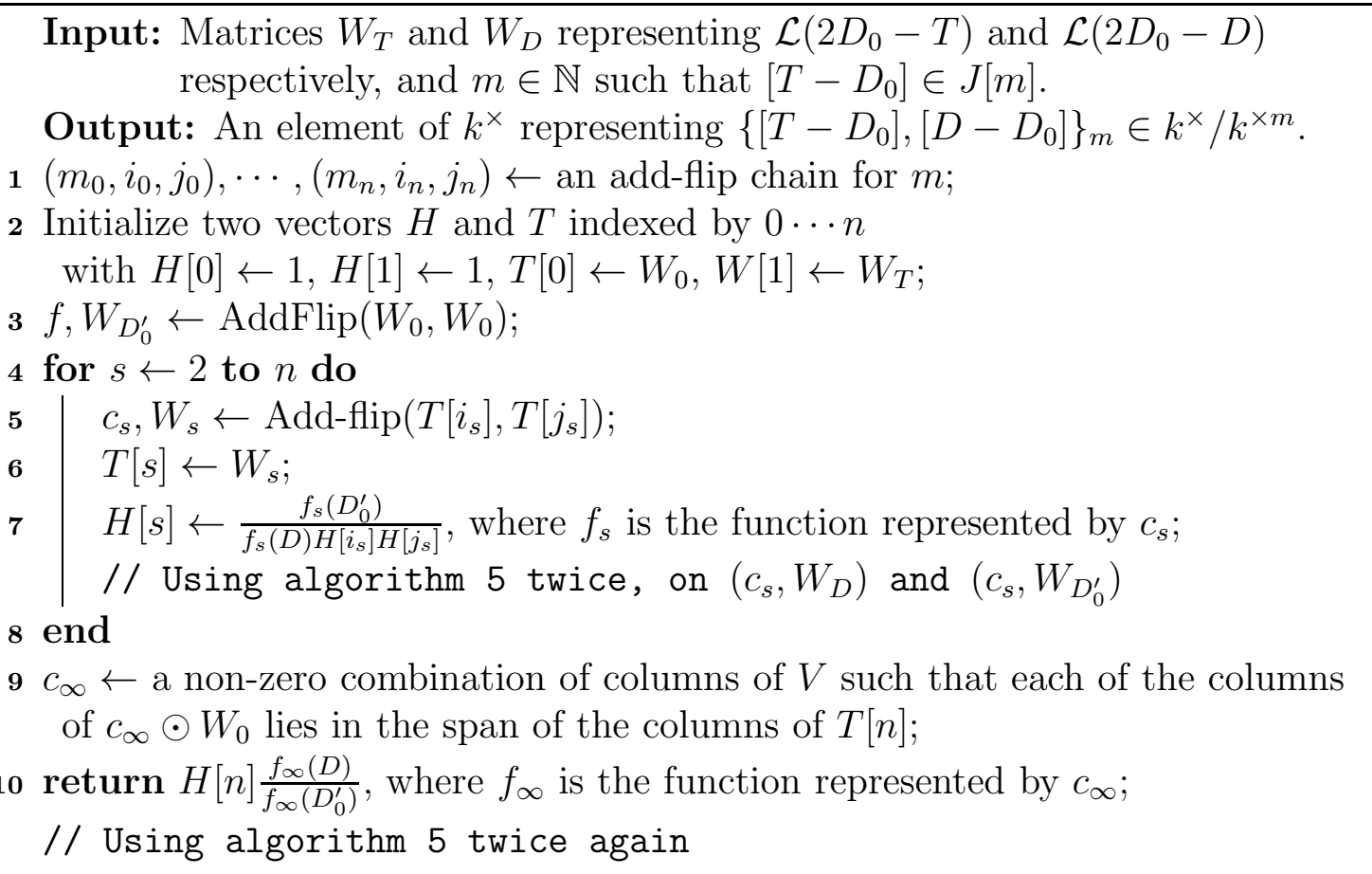

Algorithm 6: Frey-Rück pairing 
Proof. (Cf. [Bru13, p. 39] and [Mil04, section 4]) For each $s \leqslant n$, denote by $T_{s}$ the unique effective divisor of degree $d_{0}$ such that the matrix $W_{s}$ represents the space $\mathcal{L}\left(2 D_{0}-T_{s}\right)$. Clearly $T[s]=W_{T_{s}}$ and $\left[T_{s}-D_{0}\right]=m_{s}\left[T-D_{0}\right]$ for all $s$. In particular, for $s=n$ we find that $\left[T_{n}-D_{0}\right]=0$, so the space

$$
\mathcal{L}\left(D_{0}-T_{n}\right)=\left\{v \in V \mid v \mathcal{L}\left(D_{0}\right) \subset \mathcal{L}\left(2 D_{0}-T_{n}\right)\right\}
$$

is 1-dimensional. As the matrices $W_{0}$ and $T[n]=W_{n}$ represent respectively the spaces $\mathcal{L}\left(D_{0}\right)$ and $\mathcal{L}\left(2 D_{0}-T[n]\right)$, the column $c_{\infty}$ computed at line 9 represents a non-zero element $f_{\infty}$ of this space, which therefore satisfies $\left(f_{\infty}\right)=T_{n}-D_{0}$ and is unique up to multiplication by a nonzero constant.

Define inductively a sequence of functions $h_{0}, \cdots, h_{n} \in k(C)^{\times}$by $h_{0}=h_{1}=1$ and $h_{s}=1 / h_{i_{s}} h_{j_{s}} f_{s}$ for $s \geqslant 2$. Line 7 ensures that $H[s]=h_{s}\left(D-D_{0}^{\prime}\right)$ for all $s$. Besides, we have $\left(f_{s}\right)=-3 D_{0}+T_{i_{s}}+T_{j_{s}}+T_{s}$ for all $s$ by construction, so an induction on $s$ shows that

$$
\left(h_{s}\right)=m_{s} T-T_{s}-\left(m_{s}-1\right) D_{0}
$$

for all $s$. Since $\left(f_{\infty}\right)=T_{n}-D_{0}$, we get $\left(h_{n} / f_{\infty}\right)=m\left(T-D_{0}\right)$, so that

$$
\left\{\left[T-D_{0}\right],\left[D-D_{0}\right]\right\}_{m}=\left(h_{n} / f_{\infty}\right)\left(D-D_{0}^{\prime}\right)=H[n] \frac{f_{\infty}\left(D_{0}^{\prime}\right)}{f_{\infty}(D)}
$$

as $D_{0}^{\prime} \sim D_{0}$.

The complexity of this algorithm is $O\left(g^{\omega} \log m\right)$ operations in $k$, to which one should add the cost of the discrete logarithm in $\mu_{m}$ if one wants to use the linearized version of the pairing.

Remark 2.12. We have introduced the divisor $D_{0}^{\prime}$ because the functions $f_{s}$ and $f_{\infty}$, being elements of $\mathcal{L}\left(3 D_{0}\right)$, have poles at $D_{0}$, and therefore cannot be evaluated there.

Remark 2.13. This algorithm will fail if algorithm 5 fails or returns 0 . In this case, one should try again, making sure that at line 3 of algorithm 3 , the function $f$ (which is called $f_{s}$ in algorithm 6) is chosen randomly, and possibly after replacing $D$ with a linearly equivalent divisor (using the same strategy as for the construction of $D_{0}^{\prime}$ ). In practice, failure is rare since we work over a finite field large enough that the curve has enough rational points to satisfy (2.2).

Note by the way that it is important here to always choose $f$ randomly. Indeed, the add-flip algorithm 3 chooses $f$ as a nonzero element of a space which is computed as a kernel, and most computer algebra systems give kernels by bases in echelon form. As a result, if for instance we always chose the first basis vector for $f$, then some coordinates of $f$ would always be 0 ; in other words $f$ would always vanish at some fixed points, which would lead to systematic failure of algorithm 5 .

Remark 2.14. It is straightforward to modify algorithm 6 so as to compute Weil pairings if desired.

\subsubsection{Local charts and evaluation maps}

We now give a construction of Galois-equivariant rational maps $J \rightarrow \mathbb{A}^{1}$ inspired by the method for modular curves described in [Mas13, section 3.6]. In this construction, we have in mind the case where the ground field is a number field $k_{0}$ and 
where we deal with points of $J\left(\overline{k_{0}}\right)$, and our goal is to obtain a map yielding values of reasonable arithmetic height (cf [Mas13 for details). Of course, condition 2.2 will not, in general, be satisfiable if we insist that the divisor $Z$ be formed of points in $C\left(k_{0}\right)$, so we use points over a $p$-adic field $k \supset k_{0}$ instead. In other words, we actually apply Makdisi's algorithms to the base change of $C$ from $k_{0}$ to $k$.

We begin by constructing a map with values in the projective space $\mathbb{P}(V)$. For this, we assume that we are able to find effective divisors $E_{1}$ and $E_{2}$, both of degree $d_{0}-g$. Then $D_{0}-E_{1}$ has degree $g$, so given a sufficiently generic point $x \in J$, Riemann-Roch shows that there exists a generically unique effective divisor $E_{x}$ of degree $g$ such that

$$
\left[D_{0}-E_{1}-E_{x}\right]=x .
$$

Another application of Riemann-Roch then shows that the space $\mathcal{L}\left(2 D_{0}-E_{1}-E_{2}-\right.$ $E_{x}$ ) has dimension 1 , so we get a map

$$
\begin{aligned}
& J \quad \rightarrow \mathbb{P}(V) \\
& x \longmapsto \mathcal{L}\left(2 D_{0}-E_{1}-E_{2}-E_{x}\right)
\end{aligned}
$$

which is only a rational map due to the genericity assumptions on $x$.

The point of this definition is that in Makdisi's framework, there are many divisors $D$ representing a given $x \in J$, that is to say such that $\left[D-D_{0}\right]=x$. This map is thus useful to turn the representation $\mathcal{L}\left(2 D_{0}-D\right)$ of $x$ into something that does not depend on $D$, but only on $x$. Implementing it is fairly straightforward:

Input: A matrix $W_{D}$ for the space $\mathcal{L}\left(2 D_{0}-D\right)$ representing a point $x=\left[D-D_{0}\right] \in J$, and matrices $W_{1}$ and $W_{2}$ for the spaces $\mathcal{L}\left(2 D_{0}-E_{1}\right)$ and $\mathcal{L}\left(2 D_{0}-E_{2}\right)$, respectively.

Output: The value in $\mathbb{P}(V)$ of the map (2.16) at $x$.

$1 S_{1}^{\prime} \leftarrow \operatorname{DivAdd}\left(W_{D}, W_{1}\right)$;

$2 S_{1} \leftarrow \operatorname{DivSub}\left(S_{1}^{\prime}, M_{V}\right)$

3 if number of columns of $S_{1}>1$ then return FAIL;

$4 s_{1} \leftarrow$ the lone column of $S_{1} ; \quad / /\left(s_{1}\right)=-2 D_{0}+D+E_{1}+E_{x}$

$5 S_{2}^{\prime} \leftarrow \operatorname{DivSub}\left(s_{1} \odot M_{V}, W_{D}\right) ;$

$6 S_{2}^{\prime \prime} \leftarrow \operatorname{DivAdd}\left(S_{2}^{\prime}, W_{2}\right)$;

$7 S_{2} \leftarrow \operatorname{DivSub}\left(S_{2}^{\prime \prime}, M_{V}\right)$;

8 if number of columns of $S_{2}>1$ then return FAIL;

9 return $S_{2}$;

Algorithm 7: Evaluation of a rational map $J \rightarrow \mathbb{P}(V)$

Proof. The matrix $S_{1}$ represents the space $\mathcal{L}\left(2 D_{0}-D-E_{1}\right)$, which is generically of dimension 1 by Riemann-Roch. Thus $s_{1}$ is well-defined up to multiplication by a constant, and its divisor is $\left(s_{1}\right)=-2 D_{0}+D+E_{1}+E_{x}$.

Remark 2.17. We must ensure that the effective divisors $D_{0}, E_{1}$, and $E_{2}$ are actually defined over $k_{0}$ (as opposed to $k$ ) if we want the map (2.16) to be $\operatorname{Gal}\left(\overline{k_{0}} / k_{0}\right)$ equivariant. For $D_{0}$, this is not a problem in practice, since the only requirement that we need to satisfy is that $d_{0} \stackrel{\text { def }}{=} \operatorname{deg} D_{0} \geqslant 2 g+1$ (however we should try to chose $D_{0}$ so that $d_{0}$ is as close to $2 g+1$ as possible for efficiency). On the other hand, $E_{1}$ and $E_{2}$ must be of degree exactly $d_{0}-g$, and this condition can be more difficult to satisfy over a number field; but if we have to, we can take $E_{1}$ and $E_{2}$ to be defined over a finite extension of $k_{0}$, and form symmetric combinations of the values obtained for these divisors and their Galois conjugates. 
Remark 2.18. The map (2.16) may be used as a local coordinate chart on $J$, and for this purpose, $E_{1}$ and $E_{2}$ need not be defined over $k_{0}$. One should however be aware that the domain on which this map is an immersion may be smaller than its domain of definition.

We now show how to turn the map (2.16) into an $\mathbb{A}^{1}$-valued map. In [Mas13], we chose two rational points $P, Q \in C(k)$ away from the supports of $D_{0}, E_{1}$, and $E_{2}$, and we composed the map (2.16) with the map $s_{2} \longmapsto s_{2}(P) / s_{2}(Q)$, were $s_{2}$ is a nonzero element of the 1-dimensional space $\mathcal{L}\left(2 D_{0}-E_{1}-E_{2}-E_{x}\right)$. This approach was suitable for modular curves, since they have plenty of rational cusps, but is harder to adapt to a general curve; we therefore propose a different approach.

We fix a basis $v_{1}, v_{2}, \cdots$ of $V$, where the $v_{i}$ are defined over $k_{0}$ (again for Galois-equivariance reasons). For instance, if $C$ is given to us by a plane equation $f(x, y)=0$ over $k_{0}$, we will have obtained the $v_{i}$ as explicit elements of $k_{0}(x, y)$ by a Riemann-Roch space computation over $k_{0}$. We then write $s_{2}=\sum \lambda_{i} v_{i}$, where the $\lambda_{i}$ are scalars, and simply return the ratio $\lambda_{i_{1}} / \lambda_{i_{2}}$, where $i_{1}$ and $i_{2}$ are fixed indices. This is well-defined, since $s_{2}$ is well-defined up to multiplication by a constant, and straightforward to implement in Makdisi's framework. In summary, our $\mathbb{A}^{1}$ valued map is

$$
\alpha: \begin{array}{lll}
J & \rightarrow \mathbb{A}^{1} \\
x & \longmapsto & \lambda_{i_{1}} / \lambda_{i_{2}}
\end{array}
$$

where the $\lambda_{i}$ are such that $\sum_{i} \lambda_{i} v_{i}$ spans $\mathcal{L}\left(2 D_{0}-E_{1}-E_{2}-E_{x}\right)$.

The complexity of one map evaluation is $O\left(g^{\omega}\right)$ operations in $k$.

Remark 2.20. When we compute Galois representations, we will evaluate $\alpha$ at the

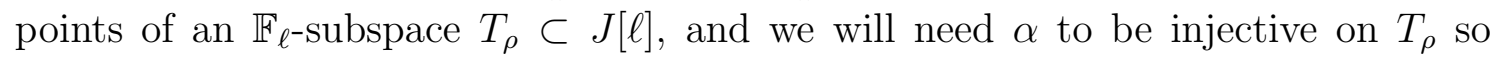
that the Galois action on its values faithfully reflects the Galois action on the points of $T_{\rho}$. Then the polynomial

$$
F(x)=\prod_{t \in T_{\rho}}(x-\alpha(t))
$$

will have coefficients in $k_{0}$, and its splitting field over $k_{0}$ will be a subfield of the field cut out by the Galois representation, and will agree with this field with high probability. We can ensure that this is indeed the case by checking that $F(x)$ is squarefree.

We must ensure that the divisors $E_{1}$ and $E_{2}$ are such that $\left[E_{1}-E_{2}\right] \notin T_{\rho}$ if $\ell \neq 2$. Indeed, the divisor of $s_{2}$ is of the form $\left(s_{2}\right)=-2 D_{0}+E_{1}+E_{2}+E_{x}+E_{x}^{\prime}$ where $E_{x}^{\prime}$ is effective of degree $g$, and (2.15) shows that

$$
x=\left[D_{0}-E_{1}-E_{x}\right]=-\left[D_{0}-E_{2}-E_{x}^{\prime}\right] .
$$

Thus if there is a $t \in T_{\rho}$ such that $\left[E_{1}-E_{2}\right]=t$, then $E_{x}^{\prime}=E_{t-x}$, so that the map $x \mapsto s_{2}$ cannot be injective on $T_{\rho}$ unless $\ell=2$.

Remark 2.21. In particular, $E_{1}$ and $E_{2}$ should be distinct. In some cases, we may be able to find a effective divisor $E_{1}$ of degree $d_{0}-g$ defined over $k_{0}$, but not a second one; in this case, we can replace the map (2.16) with the map

$$
\begin{aligned}
& J \quad \rightarrow \operatorname{Grass}_{d_{W}}(V) \\
& x \longmapsto \mathcal{L}\left(2 D_{0}-E_{1}-E_{x}\right),
\end{aligned}
$$


where $\operatorname{Grass}_{d_{W}}(V)$ is the Grassmanian of $V$ parametrising subspaces of dimension $d_{W}=d_{0}+1-g$; in other words, interrupt algorithm 7 at line 5 . Note that by definition, $E_{1}+E_{x}$ is the only effective divisor $E \geqslant E_{1}$ such that $\left[E-D_{0}\right]=x$, so that the space $\mathcal{L}\left(2 D_{0}-E_{1}-E_{x}\right)$ may be viewed as the normal form representation of $x$ (with respect ot $E_{1}$ ) in Makdisi's framework.

The advantage of this approach is that we need only one $k_{0}$-rational effective divisor of degree $d_{0}-g$, and that it requires half less computation; its disadvantage is that (2.22) now takes values in the Grassmanian of $V$, since $\operatorname{dim} \mathcal{L}\left(2 D_{0}-E_{1}-E_{x}\right)=$ $d_{W}>1$ for generic $x$. We can still use Grassmanian coordinates with respect to the basis $\left(v_{i}\right)$ of $V$ to turn (2.22) into an $\mathbb{A}^{1}$-valued map, but experiment shows that the values thus obtained have a worse arithmetic height. In what follows, we will only use (2.19).

\subsubsection{Makdisi over $p$-adic fields}

We will later apply Makdisi's algorithms over the ground field $k=\mathbb{Q}_{q}$. This means that we must be able to perform linear algebra over $k$, namely kernel and equation matrices (these are actually the same up to transposition), which is a non-trivial problem since we can only represent elements of $k$ on a computer with finite $p$-adic accuracy.

For this, we use Page's recent implementation in Pari/GP of the computation of kernels in Howell form over rings of the form $R=\mathbb{Z}_{q} / p^{e}$, where $\mathbb{Z}_{q}$ is the ring of integers of $\mathbb{Q}_{q}$ and $e \in \mathbb{N}$. Recall that the Howell form of an $R$-submodule $M$ of $R^{d}(d \in \mathbb{N})$ is a canonical generating set of this module, which is essentially characterized by the fact that these generators are in reduced echelon form, and that for all $d^{\prime} \leqslant d$, each element of $M$ whose first $d^{\prime}$ entries are 0 is an $R$-combination of the generators whose first $d^{\prime}$ entries are 0; cf. [How86] or [SM98] (beware that these references consider row spans, whereas we deal with column spans in this article). The following result shows that if we consider an approximation up to $O\left(p^{e}\right)$ of a matrix $A$ with coefficients in $\mathbb{Z}_{q}$ which has "good reduction", i.e. whose rank does not decrease after reduction $\bmod p$, then we can compute a kernel matrix of $A$ with the same accuracy and satisfying the same conditions, and thus use Makdisi's algorithms without loss of $p$-adic accuracy.

Theorem 2.23. Let $k$ be a non-Archimedian local field with ring of integers $\mathcal{O}$, uniformizer $\varpi$ and residue field $\kappa$, and let $A$ be an $m \times n$ matrix with coefficients in $\mathcal{O}$, representing a linear map $T: \mathcal{O}^{n} \rightarrow \mathcal{O}^{m}$. Let $e \in \mathbb{N}$, and let $K_{e}$ be the kernel of the induced map $T_{e}:\left(\mathcal{O} / \varpi^{e}\right)^{n} \rightarrow\left(\mathcal{O} / \varpi^{e}\right)^{m}$. If $\operatorname{rk}_{k}(A)=\operatorname{rk}_{\kappa}(A \bmod \varpi)$, then the Howell generators of $K_{e}$ that are nonzero $\bmod \varpi$ form an approximation $\bmod \varpi^{e}$ of an $\mathcal{O}$-basis of $\operatorname{ker} T$.

Remark 2.24. There can be Howell generators of $K_{e}$ that are $0 \bmod \varpi$, as demon-

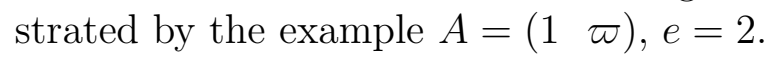

Proof. Let us write $K_{\infty}=\operatorname{ker} T$, and $K_{k}$ for the kernel of the induced map $k^{n} \rightarrow k^{m}$, so that $K_{\infty}=K_{k} \cap \mathcal{O}^{n}$. Since $\mathcal{O}$ is a PID, we can consider an $\mathcal{O}$-basis $h_{1}, \cdots, h_{d} \in$ $\mathcal{O}^{n}$ of $K_{\infty}$ in Hermite normal form.

We claim that the $h_{i}$ are linearly independent $\bmod \varpi$. Indeed, let $\lambda_{i} \in \mathcal{O}$, and let $x=\sum_{i} \lambda_{i} h_{i} \in K_{\infty}$. If $x \equiv 0 \bmod \varpi$, say $x=\varpi y$ for some $y \in \mathcal{O}^{n}$, then $y \in \mathcal{O}^{n} \cap K_{k}=K_{\infty}$, which shows that the $\lambda_{i}$ are all $0 \bmod \varpi$ since the $h_{i}$ are linearly independent over $k$. 
We can then prove by induction on $e$ that the $h_{i}$ generate $K_{e}$. Indeed, for $e=1$ this follows from the above and the fact that the rank of $A$ does not decrease after reduction mod $\varpi$. Suppose this is true for $e$, and let $x \in \mathcal{O}^{n}$ be such that $x \bmod \varpi^{e+1}$ lies in $K_{e+1}$. Then $x \bmod \varpi^{e}$ lies in $K_{e}$, so there exist $\lambda_{i} \in \mathcal{O}$ and $y \in \mathcal{O}^{n}$ such that $x=\sum_{i} \lambda_{i} h_{i}+\varpi^{e} y$; therefore

$$
0 \equiv A x=\varpi^{e} A y \bmod \varpi^{e+1}
$$

Dividing by $\varpi^{e}$ shows that $y \bmod \varpi$ lies in $K_{1}$, and is thus in the $\mathcal{O}$-span of the $h_{i}$, and so is $x$.

It follows that the Howell form of $K_{e}$ agrees with that of the $h_{i} \bmod \varpi^{e}$; however the $h_{i}$ are in echelon form, so they are part of their Howell form mod $\varpi^{e}$, and the other vectors must be linear combinations of them that are $0 \bmod \varpi$.

Remark 2.25. It is shown in [SM98] that the Howell form of ker $A \bmod p^{e}$ can be computed with the same complexity as if $R=\mathbb{Z}_{q} / p^{e}$ were a field, namely $O\left(n^{\omega}\right)$ operations in $R$ for $A$ of size $n \times n$. Therefore, we can execute the algorithms presented in this section over $R$ with the same number of operations in $R$ as if $R$ were a field.

\subsubsection{Galois action}

We now suppose that the curve $C$ is defined over $\mathbb{Q}_{p}$ (in practice, it is actually defined over $\mathbb{Q}$ ), and that we have a matrix $W_{D}$ with coefficients in $\mathbb{Z}_{q} / p^{e}$ representing a point $x \in J\left(\mathbb{Z}_{q} / p^{e}\right)$ for some $e \in \mathbb{N}$ (in particular, if $e=1$ then $W_{D}$ represents a point in $J\left(\mathbb{F}_{q}\right)$ ). We would like to compute a matrix $W_{D^{\prime}}$ representing $x^{\Phi}$ to the same p-adic accuracy, where $\Phi \in \operatorname{Gal}\left(\mathbb{Q}_{q} / \mathbb{Q}_{p}\right)$ is the Frobenius. This is easy, provided that the divisors $D_{0}$ and $Z$ are defined over $\mathbb{Q}_{p}$, and that we know how $\Phi$ acts on the support of $Z$ (in practice this information should be recorded at the creation of $Z$ ).

\footnotetext{
Input: A matrix $W_{D}$ representing a point $x \in J\left(\mathbb{Q}_{q}\right)$.

Output: A matrix $W_{D^{\prime}}$ representing the point $x^{\Phi} \in J\left(\mathbb{Q}_{q}\right)$.

$1 W \leftarrow W_{D}^{\Phi}$

2 Permute the rows of $W$ by the permutation induced by $\Phi^{-1}$ on $Z$;

3 return $W$;
}

\section{Algorithm 8: Frobenius}

Proof. Recall that the $j$-th column of $W_{D}$ represents a function $w_{j}$ such that the $w_{j}$ form $\mathbb{Q}_{q}$-basis of the space $\mathcal{L}\left(2 D_{0}-D\right)$ representing $x$, in that the $i$-th row of $W_{D}$ contains the value of the $w_{j}$ at the $i$-th point in the support of $Z$. Since $x=\left[D-D_{0}\right]$, we have $x^{\Phi}=\left[D^{\Phi}-D_{0}\right]$, so $x^{\Phi}$ is represented by $\mathcal{L}\left(2 D_{0}-D^{\Phi}\right)=\mathcal{L}\left(2 D_{0}-D\right)^{\Phi}$, but $v^{\Phi}(P)=\left(v\left(P^{\Phi^{-1}}\right)\right)^{\Phi}$ for all $P \in C\left(\mathbb{Q}_{q}\right)$.

The complexity of this algorithm is of course $O\left(g^{2}\right)$ operations in $\mathbb{Q}_{q}$.

Remark 2.26. We use a model for $\mathbb{Q}_{q}$ of the form $\mathbb{Q}_{p}(\theta) / T(\theta)$, where $T(x) \in \mathbb{Z}[x]$ is a lift of an irreducible polynomial over $\mathbb{F}_{p}$. In this model, every element of $\mathbb{Q}_{q}$ is of the form $\sum_{i} c_{i} \theta^{i}$ with $c_{i} \in \mathbb{Q}_{p}$, and the image of such an element by $\Phi$ is simply $\sum_{i} c_{i} \theta^{i}$, where $\theta^{\prime}$ is the unique root of $T(x)$ congruent to $\theta^{p} \bmod p$. 


\section{Differentiation of linear algebra operations}

Let us now suppose that the ground field is a finite extension $k$ of $\mathbb{Q}_{p}$ with ring of integers $\mathcal{O}$ and uniformizer $\varpi$ (in practice, $k=\mathbb{Q}_{q}, \mathcal{O}=\mathbb{Z}_{q}$, and $\varpi=p$ ). Makdisi's algorithms rely on linear algebra; in particular, they frequently involve the computation of a kernel matrix or of an equation matrix (a.k.a. left kernel) of a given matrix $A$ with coefficients in $\mathcal{O}$. The goal of this section is to determine how these deform under $\varpi$-adically small perturbations of $A$.

However, the problem thus presented is ill-defined, since a given vector space (in this case, the right or left kernel of $A$ ) admits many different bases in general. We therefore start by rigidifying the notions of kernel matrix and of equation matrix defined at page 5. For simplicity, we restrict ourselves to the case where the matrix $A$ has full rank.

Let us treat the case of equation matrices first, since only this case will be used later. We assume that we have set a rule that, given a matrix ${ }_{n} A_{r}$ (this notation means that $A$ has $n$ rows and $r$ columns) with coefficients in $\mathcal{O}$ and whose reduction mod $\varpi$ has rank $r$, assigns to it a supplement matrix, that is to say a matrix ${ }_{n} S_{n-r}$ with coefficients in $\mathcal{O}$ such that the augmented matrix $A^{+}=\left({ }_{n} A_{r} \mid{ }_{n} S_{n-r}\right)$ is invertible over $\mathcal{O}$, and which only depends on the reduction of $A \bmod \varpi$. For instance, we can impose that the $j$-th column of $S$ has a 1 at row $i_{j}$ and 0 everywhere else, in such a way that $i_{1}<\cdots<i_{n-r}$ and that the $i_{j}$ are as small as possible with respect to some total ordering; this is what $[$ Pari/GP $\mid$ 's function matsupplement does.

Let us decompose the inverse $B=\left(A^{+}\right)^{-1}$ of $A^{+}$over $\mathcal{O}$ as

$$
B=\left(\frac{{ }_{r} F_{n}}{{ }_{n-r} E_{n}}\right) \text {. }
$$

We have

$$
I_{n}=B A^{+}=\left(\begin{array}{c|c}
{ }_{r} F A_{r} & { }_{r} F S_{n-r} \\
\hline{ }_{n-r} E A_{r} & { }_{n-r} E S_{n-r}
\end{array}\right),
$$

whence the relations $F A=I_{r}, F S={ }_{r} 0_{n-r}, E A={ }_{n-r} 0_{r}$, and $E S=I_{n-r}$. In particular, the rows of $E$ are linearly independent (both over $k$ and over its residue field), so that $E$ is an equation matrix for $A$. We call $E$ the equation matrix of $A$, and denote it by $E=\operatorname{Eqn}(A)$, the rule determining $S$ being implicit. Besides, we call $F$ the $F$-complement of $A$.

The advantage of this definition is that it is easy to differentiate $\operatorname{Eqn}(A)$ with respect to $A$. Indeed, let ${ }_{n} H_{r}$ be a matrix of the same size as $A$ and whose entries lie in $\varpi^{e} \mathcal{O}$ for some integer $e \geqslant 1$. The matrix $A+H$ is congruent to $A \bmod \varpi$, so the augmented matrix $(A+H \mid S)$ is still invertible (with the same $S$ ) over $\mathcal{O}$, whence $(A+H)^{+}=A^{+}+H^{0}$ where $H^{0}=\left({ }_{n} H_{r} \mid{ }_{n} 0_{n-r}\right)$. Therefore

$$
\begin{aligned}
\left((A+H)^{+}\right)^{-1} & =B-B H^{0} B+O\left(\varpi^{2 e}\right) \\
& =\left(\frac{{ }_{r} F_{n}}{{ }_{n-r} E_{n}}\right)-\left(\frac{{ }_{r} F_{n}}{{ }_{n-r} E_{n}}\right)\left({ }_{n} H_{r} \mid{ }_{n} 0_{n-r}\right)\left(\frac{{ }_{r} F_{n}}{{ }_{n-r} E_{n}}\right)+O\left(\varpi^{2 e}\right) \\
& =\left(\frac{{ }_{r} F_{n}}{{ }_{n-r} E_{n}}\right)-\left(\frac{{ }_{r} F H F_{n}}{{ }_{n-r} E H F_{n}}\right)+O\left(\varpi^{2 e}\right),
\end{aligned}
$$

which shows that

$$
\operatorname{Eqn}(A+H)=\operatorname{Eqn}(A)-\operatorname{Eqn}(A) H F+O\left(\varpi^{2 e}\right)
$$


where $F$ is the F-complement of $A$ and $O\left(\varpi^{2 e}\right)$ denotes a matrix whose entries lie in $\varpi^{2 e} \mathcal{O}$.

The case of kernels, being the transpose of the previous case, is similar: given ${ }_{r} A_{n}$ with coefficients in $\mathcal{O}$ and whose reduction mod $\varpi$ has rank $r$, we set

$$
A^{+}=\left(\frac{{ }_{r} A_{n}}{{ }_{n-r} S_{n}}\right)
$$

where $S$ is determined by similar rules so that $A^{+}$is invertible, and then if

$$
\left(A^{+}\right)^{-1}=\left({ }_{n} L_{r} \mid{ }_{n} K_{n-r}\right)
$$

then $K$ is a kernel matrix of $A$, which we denote by $K=\operatorname{Ker}(A)$ with a capital $\mathrm{K}$; besides we have

$$
\operatorname{Ker}(A+H)=\operatorname{Ker}(A)-L H \operatorname{Ker}(A)+O\left(\varpi^{2 e}\right)
$$

for $H$ of the same size as $A$ and with coefficients in $\varpi^{e} \mathcal{O}$.

Remark 3.3. In the next section, we will work with matrices with coefficients in $R=\mathbb{Z}_{q} / p^{2 e}$, and our perturbation matrices $H$ will have coefficients in $I=p^{e} R$. Since $I^{2}=0$, all the $O\left(\varpi^{2 e}\right)$ terms will vanish in $R$.

\section{$4 \quad p$-adic lifting of points of $J$}

In this section, we suppose that we are given a matrix $W$ with coefficients in $\mathbb{Z}_{q} / p^{e}$ representing a point of $J\left(\mathbb{Z}_{q} / p^{e}\right)$ (in other words, a point of $J\left(\mathbb{Q}_{q}\right)=J\left(\mathbb{Z}_{q}\right)$ with absolute accuracy $O\left(p^{e}\right)$ ) for some $e \in \mathbb{N}$, whereas the data representing $J$ itself have been precomputed with accuracy at least $O\left(p^{2 e}\right)$ (meaning that the matrices $K_{V_{3}}, M_{V}$, etc., have coefficients in $\mathbb{Z}_{q} / p^{e^{\prime}}$ where $\left.e^{\prime} \geqslant 2 e\right)$. Our goal is to find a deformation of $W$ over $\mathbb{Z}_{q} / p^{2 e}$ which represents a point of $J\left(\mathbb{Z}_{q} / p^{2 e}\right)$. Once we have solved this problem, we will be able to lift quadratically a representation of a point in $\bar{x} \in J\left(\mathbb{F}_{q}\right)$ (given by a matrix for $W_{D}$ with coefficients in $\mathbb{F}_{q}$ ) to a representation to arbitrary $p$-adic accuracy of a point of $\widetilde{x} \in J\left(\mathbb{Q}_{q}\right)$ which reduces to $\bar{x} \bmod p$.

Our approach consists in determining how a deformation of the input of the membership test (algorithm 4) perturbs the execution of this algorithm. Let us introduce some notation: given a commutative $\operatorname{ring} R$ and a column vector $c \in R^{n_{Z}}$, we will denote by $\Delta_{c}$ the diagonal matrix of size $n_{Z}$ and coefficients in $R$ whose diagonal is formed of the coefficients of $c$. Also define $r=n_{Z}-d_{W}$.

Let $S$ be a matrix of size $n_{Z} \times d_{W}$ and coefficients in $\mathbb{Z}_{q}$, whose columns $s_{i} \in \mathbb{Z}_{q}^{n_{Z}}$ are independent $\bmod p$ and form a basis of a $\mathbb{Q}_{q}$-subspace of $V$ of dimension $d_{W}$ (this is the case in particular if $S$ is an arbitrary lift of $W$ still representing a subspace of $V$, in view of the conventions established in remark 2.4). An analysis of algorithm 4 shows that if we called this algorithm on $S$, then the matrix $W^{\prime}$ introduced at line 2 would be computed as the kernel of the matrix

$$
\left(\begin{array}{c}
\frac{K_{V_{3}}}{\vdots} \\
\operatorname{Eqn}\left(s_{1} \odot M_{V}\right) \Delta_{s_{m}} \\
\vdots
\end{array}\right)
$$


where $m$ ranges from 1 to $d_{W}$ and where we assume without loss of generality that the $w$ chosen at line 1 is $s_{1}$. Indeed, the block of equations $K_{V_{3}}$ describes the column vectors $c \in \mathbb{Q}_{q}^{n_{Z}}$ representing elements of $V_{3}=\mathcal{L}\left(3 D_{0}\right)$, whereas for each $m$ the block of equations $\operatorname{Eqn}\left(s_{1} \odot M_{V}\right) \Delta_{s_{m}}$ describes the $c$ such that $s_{m} \odot c$ represents an element of $f_{1} V=f_{1} \mathcal{L}\left(2 D_{0}\right)$, where $f_{1}$ is the function represented by $s_{1}$. In particular, for $m=1$ this block is redundant with $K_{V_{3}}$ since $D_{0} \geqslant 0$; we therefore define

$$
K(S)=\left(\begin{array}{c}
\frac{K_{V}}{\vdots} \\
\operatorname{Eqn}\left(s_{1} \odot M_{V}\right) \Delta_{s_{m}} \\
\vdots
\end{array}\right)
$$

where $m$ ranges from 2 to $d_{W}$. Algorithm 4 shows that $S$ represents an element of $J\left(\mathbb{Q}_{q}\right)$ if and only if the $\mathbb{Q}_{q}$-rank of $K(S)$ is $r$ and not more.

Since by assumption the matrix $W$ represents a point of $J\left(\mathbb{Z}_{q} / p^{e}\right)$, the matrix $K(W)$ is thus the reduction $\bmod p^{e}$ of a matrix with coefficients in $\mathbb{Z}_{q}$ and $\mathbb{Q}_{q^{-}}$ rank $r$.

Let now $\widetilde{W}$ be a arbitrary lift of $W$ to $\mathbb{Z}_{q} / p^{2 e}$ which still represents a subspace of $V$. For instance, such a lift can be obtained by finding a matrix $U$ with coefficients in $\mathbb{Z}_{q} / p^{e}$ such that $W=M_{V} U$, and taking $\widetilde{W}=M_{V} \widetilde{U}$ where $\widetilde{U}$ is an arbitrary lift of $U$ to $\mathbb{Z}_{q} / p^{2 e}$. The matrix $K(\widetilde{W})$ is then a lift of $K(W)$ to $\mathbb{Z}_{q} / p^{2 e}$, and $\widetilde{W}$ represents a point $\widetilde{x} \in J\left(\mathbb{Z}_{q} / p^{2 e}\right)$ if and only if $K(\widetilde{W})$ is the reduction $\bmod p^{2 e}$ of a matrix with coefficients in $\mathbb{Z}_{q}$ and of $\mathbb{Q}_{q}$-rank $r$ (and $\widetilde{x}$ is then necessarily a lift of the point $x \in J\left(\mathbb{Z}_{q} / p^{e}\right)$ represented by $\left.W\right)$.

Enforcing this rank condition in terms of vanishing of minors would be inefficient; instead we use the following method.

Lemma 4.1. Let ${ }_{m} M_{n}=\left(\begin{array}{c|c}{ }_{r} A_{r} & { }_{r} B_{n-r} \\ \hline{ }_{m-r} C_{r} & { }_{m-r} D_{n-r}\end{array}\right)$ be an $m \times n$ matrix with coefficients in a field such that the $r \times r$ block $A$ is invertible. Then $\operatorname{rk} M \geqslant r$, with equality iff.

$$
D-C A^{-1} B={ }_{m-r} 0_{n-r} .
$$

Proof. The matrix ${ }_{n} P_{n}=\left(\begin{array}{c|c}I_{r} & -A^{-1} B \\ \hline 0 & I_{n-r}\end{array}\right)$ is clearly invertible, and

$$
M P=\left(\begin{array}{c|c}
A & 0 \\
\hline C & D-C A^{-1} B
\end{array}\right) .
$$

Let $\bar{K}$ be the reduction of $K(\widetilde{W}) \bmod p$. It has coefficients in $\mathbb{F}_{q}$, and does not depend on the lift $\widetilde{W}$ of $W$. Since $W$ represents a point of $J\left(\mathbb{Z}_{q} / p^{e}\right)$ with $e \geqslant 1$, the $\mathbb{F}_{q}$-rank of $\bar{K}$ is exactly $r$; indeed $\bar{K}=K(\bar{W})$, where $\bar{W}$ is the reduction of $W$ $\bmod p$, which does represents a point of $J\left(\mathbb{F}_{q}\right)$. Therefore, $\bar{K}$ has an invertible minor of size $r$. Possibly after permuting the rows and columns of $K(\widetilde{W})$, we may assume that the top-left $r \times r$ block of $\bar{K}$ is invertible over $\mathbb{F}_{q}$; we will suppose here that this is directly the case for the clarity of exposition. Then the top left $r \times r$ block of $K(\widetilde{W})$ is invertible over $\mathbb{Z}_{q} / p^{2 e}$. Splitting

$$
K(\widetilde{W})=\left(\begin{array}{l|l}
A & B \\
\hline C & D
\end{array}\right)
$$


as in lemma 4.1, we have therefore established that

$$
\widetilde{W} \text { represents a point of } J\left(\mathbb{Z}_{q} / p^{2 e}\right) \Longleftrightarrow D-C A^{-1} B=0 \bmod p^{2 e} .
$$

Furthermore, we can determine how $K(\widetilde{W})$ deforms with $\widetilde{W}$, thanks to the formulas established in section 3 . Indeed, let $\widetilde{w}_{1}, \cdots, \widetilde{w}_{d_{W}}$ be the columns of $\widetilde{W}$, so that

$$
K(\widetilde{W})=\left(\begin{array}{c}
K_{V} \\
\vdots \\
\operatorname{Eqn}\left(\widetilde{w}_{1} \odot M_{V}\right) \Delta_{\widetilde{w}_{m}} \\
\vdots
\end{array}\right)
$$

If we replace $\widetilde{W}$ with a deformation $\widetilde{W}+M_{V} H$ still representing a subspace of $V$, where $H$ is a matrix with coefficients in $p^{e} \mathbb{Z}_{q} / p^{2 e}$ whose columns we denote by $h_{1}, \cdots, h_{d_{W}}$, then for each $m$, the column $\widetilde{w}_{m}$ gets replaced by $\widetilde{w}_{m}+M_{V} h_{m}$, so $\Delta_{\widetilde{w}_{m}}$ gets shifted by $\Delta_{M_{V} h_{m}}$; whereas $\widetilde{w}_{1} \odot M_{V}=\Delta_{\widetilde{w}_{1}} M_{V}$ gets shifted by $\Delta_{M_{V} h_{1}} M_{V}$, so that $\operatorname{Eqn}\left(\widetilde{w}_{1} \odot M_{V}\right)$ gets shifted by $-\operatorname{Eqn}\left(\widetilde{w}_{1} \odot M_{V}\right) \Delta_{M_{V} h_{1}} M_{V} F$ by (3.1), where $F$ is the F-complement of $\operatorname{Eqn}\left(\widetilde{w}_{1} \odot M_{V}\right)$ as defined in section 3. Thus in total, $K(\widetilde{W})$ gets shifted by

$$
\left(\begin{array}{c}
\frac{0}{\vdots} \\
\operatorname{Eqn}\left(\widetilde{w}_{1} \odot M_{V}\right)\left(\Delta_{M_{V} h_{m}}-\Delta_{M_{V} h_{1}} M_{V} F \Delta_{\widetilde{w}_{m}}\right) \\
\vdots
\end{array}\right) .
$$

It is therefore easy to see how the blocks $A, B, C$, and $D$ deform in terms of the $h_{m}$, and thus to deduce linear equations that the $h_{m}$ must satisfy for $D-C A^{-1} B$ to vanish $\bmod p^{2 e}$.

These observations translate into the following algorithm: 
Input: A matrix $W$ over $\mathbb{Z}_{q} / p^{e}$ representing a point in $J\left(\mathbb{Z}_{q} / p^{e}\right)$.

Output: A matrix $\widetilde{W} \equiv W \bmod p^{e}$ over $\mathbb{Z}_{q} / p^{2 e}$ representing a lift of this point to $J\left(\mathbb{Z}_{q} / p^{2 e}\right)$.

$/ /$ Lift $W$ so that it still represents a subspace of $V$

$1 K \leftarrow$ kernel of the horizontal concatenation $\left(W \mid M_{V}\right) \bmod p^{e}$;

2 Drop the first $d_{W}$ rows of $K$ and lift the rest to $\mathbb{Z}_{q} / p^{2 e}$;

3 $\widetilde{W} \leftarrow M_{V} K \bmod p^{2 e}$

$4 w_{1}, \cdots, w_{d_{W}} \leftarrow$ the columns of $\widetilde{W}$;

// Save on number of variables

$5 I \leftarrow$ a subset of $\left\{1, \cdots, n_{Z}\right\}$ of size $d_{W}$ such that the corresponding rows of $W$ form an invertible matrix;

$6 V_{0} \leftarrow$ a matrix representing the subspace $\left\{v \in V \mid v\left(P_{i}\right)=0 \forall i \in I\right\}$ of $V$, where the $P_{i}$ are as in section 2 ;

$7 v_{1}, \cdots, v_{d_{0}} \leftarrow$ column vectors of $V_{0}$;

// Evaluate $D-C A^{-1} B$

$8 E, F \leftarrow$ equation matrix and F-complement of $w_{1} \odot M_{V}$;

$9 K \leftarrow$ stack of $K_{V}$ and of the $E \Delta_{w_{m}}$ for $2 \leqslant m \leqslant d_{W}$;

$10 A, B, C, D \leftarrow$ splitting of $K$ as in lemma 4.1,

$11 R \leftarrow\left(D-C A^{-1} B\right) / p^{e} \bmod p^{e}$;

// Precompute some data

12 for $m \leftarrow 2$ to $d_{W}$ do

$13 \mid F_{m} \leftarrow-M_{V} F \Delta_{w_{m}} \bmod p^{e}$;

14 end

$/ /$ See the effect of deforming $w_{j}$ by $p^{e} v_{i}$ for each $j \leqslant d_{W}, i \leqslant d_{0}$

15 for $i \leftarrow 1$ to $d_{0}$ do

$16 \quad E_{i} \leftarrow E \Delta_{v_{i}} \bmod p^{e}$;

$17 \quad$ for $j \leftarrow 1$ to $d_{W}$ do

$18 \quad$ if $j=1$ then

$19 \quad \mid k \leftarrow$ a matrix with the same shape as $K$, formed by stacking a

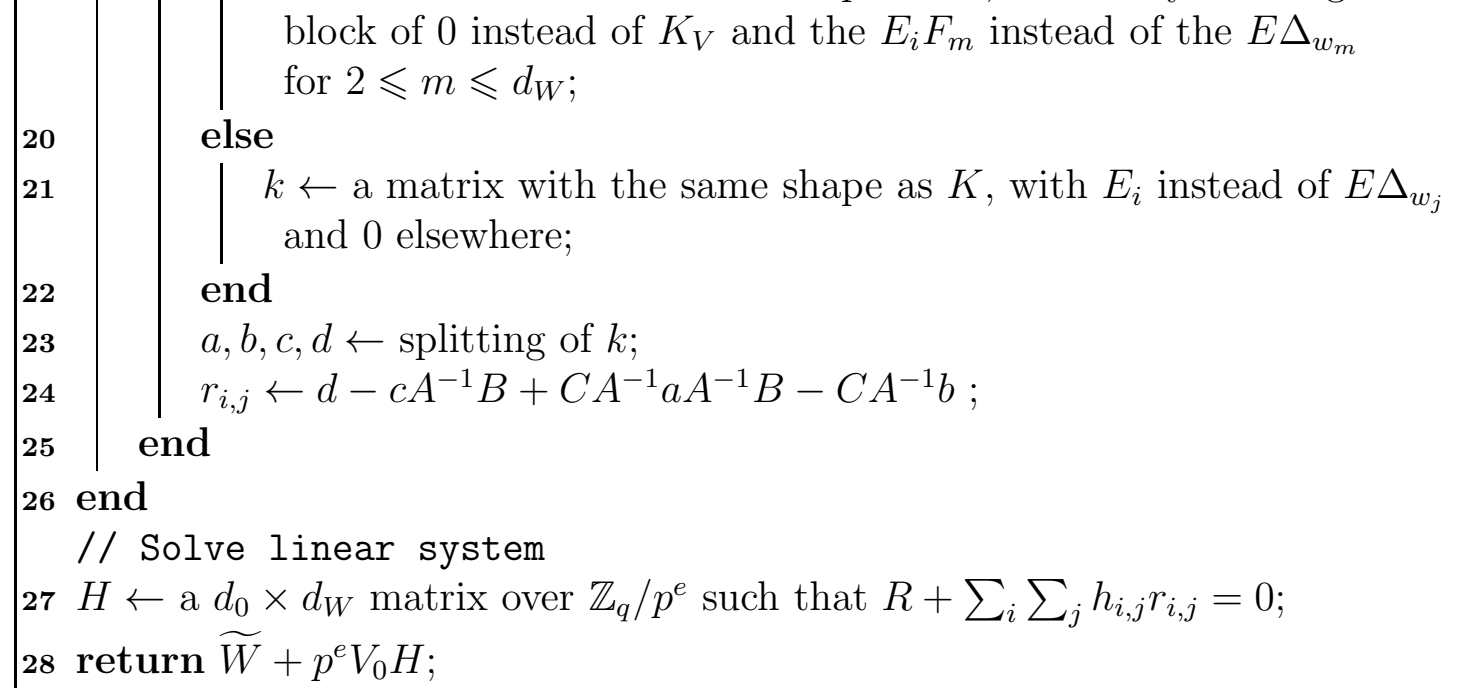

Algorithm 9: Lift 
Proof. We begin by finding a lift $\widetilde{W}$ of $W$ to $\mathbb{Z}_{q} / p^{2 e}$ which still represents a subspace of $V$; this goal is attained at line 3 .

We are then looking for a matrix $H^{\prime}$ of the same size as $W$ and with coefficients in $p^{e} \mathbb{Z}_{q} / p^{2 e} \mathbb{Z}_{q}$ such that $\widetilde{W^{\prime}}=\widetilde{W}+H^{\prime}$ satisfies criterion (4.2). In particular this lift $\widetilde{W^{\prime}}$ must also represent a subspace of $V$, so we actually look for $H^{\prime}$ of the form $H^{\prime}=M_{V} H$ for some matrix $H$ with coefficients in $p^{e} \mathbb{Z}_{q} / p^{2 e} \mathbb{Z}_{q}$.

Given a matrix $M$ with $n_{Z}$ rows, write $M_{I}$ for the matrix formed by the rows of $M$ indexed by $I$, where $I$ is defined at line 5. The matrices $\widetilde{W}_{I}$ and $\widetilde{W}_{I}^{\prime}$ are invertible over $\mathbb{Z}_{q} / p^{2 e}$ by definition of $I$. Therefore there exists an invertible matrix $P$ (which is congruent to the identity $\bmod p^{e}$ ) such that $\widetilde{W_{I}^{\prime}} P=\widetilde{W}_{I}$. Since the matrices $\widetilde{W}^{\prime} P$ and $\widetilde{W^{\prime}}$ represent two bases of the same subspace of $V$ ( $P$ being the change-ofbasis matrix), we may impose that the rows of $\widetilde{W}$ indexed by $I$ remain unchanged, i.e. that $H^{\prime}$ be actually of the form $V_{0} H$ for some $H$. This reduces the number of unknowns and thus improves the efficiency of the algorithm. Note that the number of columns of $V_{0}$ is $\operatorname{dim} V-\operatorname{dim} W=\operatorname{codim}_{V} W=d_{0}$.

We then construct the matrix $K=K(\widetilde{W})$, and split it into blocks $A, B, C, D$ as explained above. In view of (4.2), we have $D-C A^{-1} B=0$ at least $\bmod p^{e}$, so the division defining $R$ is exact.

Next, in the double loop we determine for each $i$ and $j$ how deforming $\widetilde{W}$ by adding $p^{e} v_{i}$ to its $j$-th column perturbs $R$ thanks to (4.3): in each case, we compute $k$ such that $K$ shifts by $p^{e} k$, and deduce $r_{i, j}$ such that $R$ shifts by $r_{i, j}$.

It then only remains to solve a linear system over $\mathbb{Z}_{q} / p^{e}$ so as to find a linear combination of the $r_{i, j}$ killing $R$, and to perform the corresponding change on $\widetilde{W}$.

The complexity of this algorithm is dominated by these last two steps. The matrices $A$ and $B$ have size $O(g) \times O(g)$, whereas $C$ and $D$ have size $O\left(g^{2}\right) \times O(g)$, so the matrix computations performed at line 24 require $O\left(g^{\omega+1}\right)$ operations in $\mathbb{Z}_{q} / p^{e}$. In view of remark 4.5 below, the complexity of this algorithm is thus $O\left(g^{\omega+3}\right)$ operations in $\mathbb{Z}_{q} / p^{O(e)}$.

Remark 4.4. The matrices $E_{i}$ and $F_{m}$ are precomputed for efficiency reasons. Besides, multiplications by matrices of the type $\Delta_{c}$ should not be performed literally but rather by rescaling the rows or columns as appropriate.

Remark 4.5. The tangent space of $J$ has dimension $g$, and the fibers of the map

$$
\begin{array}{ccc}
\operatorname{Eff}^{d_{0}}(C) & \longrightarrow & J \\
D & \longmapsto & {\left[D-D_{0}\right]}
\end{array}
$$

have dimension $d_{0}-g$. Since we have rigidified the situation by taking $H^{\prime}$ of the form $V_{0} H$, the linear system that we solve at line 27 has a solution space of dimension $d_{0}=O(g)$. But the matrices $K(\widetilde{W})$ and $R$ have size $O\left(g^{2}\right) \times O(g)$, so this system has $O\left(g^{3}\right)$ equations in $O\left(g^{2}\right)$ unknowns. It is thus very redundant, and a lot of time can be saved by solving $O\left(g^{2}\right)$ random combinations of its equations and checking that we have indeed obtained a solution (e.g. thanks to algorithm 41), instead of solving it directly. Without this, the complexity would be higher than $O\left(g^{\omega+3}\right)$.

Remark 4.6. Even though the performance of our implementation is quite satisfactory (cf. section 7), it is unfortunate that the complexity of our lifting algorithm is $O\left(g^{\omega+3}\right)$ whereas Makdisi's algorithms perform arithmetic in $J$ in only $O\left(g^{\omega}\right)$. In future work, we plan to improve the complexity of our lifting algorithm, by using the asymptotically faster membership test described in Proposition/Algorithm 4.12 of [KM07. 


\section{$5 \quad p$-adic lifting of torsion points}

Let $m \in \mathbb{N}$, and let $W$ be a $n_{Z} \times d_{W}$ matrix with coefficients in $\mathbb{F}_{q}$ representing an $m$-torsion point $x \in J\left(\mathbb{F}_{q}\right)[m]$. Thanks to algorithm 9 presented in the previous section, we can find a lift $\widetilde{W}$ of $W$ representing a lift $\widetilde{x} \in J\left(\mathbb{Q}_{q}\right)$ to arbitrary $p$-adic accuracy; however this point $\widetilde{x}$ will not in general be $m$-torsion. The purpose of this section is to explain how to modify algorithm 9 so that the output $\widetilde{W}$ represents a point of $J\left(\mathbb{Q}_{q}\right)$ which is still $m$-torsion.

In this section and in the next one, whenever $n \in \mathbb{Z}$ and $W$ is a matrix representing a point $x \in J$, we will denote by $[n] W$ a matrix representing $[n] x \in J$ obtained by repeated use of algorithm 3 .

For simplicity, we will assume that $m$ is coprime to $p$; indeed the discussion about formal groups below shows that this ensures that the $m$-torsion lift of $x$ to $J\left(\mathbb{Q}_{q}\right)$ is unique. In practice, we will take $m=\ell$, a prime distinct from $p$.

We actually present two methods: the first one is more efficient when the $p$-adic accuracy is still low, whereas the second one should be used when $p$-adic accuracy becomes higher.

\subsection{Method 1: Killing the kernel of reduction}

Let $\mathfrak{p}=p \mathbb{Z}_{q}$ be the maximal ideal of $\mathbb{Z}_{q}$, and for each $e \in \mathbb{N}$, let us denote by $J_{e}$ the kernel of the reduction map $J\left(\mathbb{Q}_{q}\right) \longrightarrow J\left(\mathbb{Z}_{q} / p^{e}\right)$. For instance $J_{1}$ is the kernel of $J\left(\mathbb{Q}_{q}\right) \longrightarrow J\left(\mathbb{F}_{q}\right)$.

Since $J$ has good reduction at $p$, and since $\mathbb{Q}_{q}$ is unramified, the theory of formal groups (cf. for instance [Sil09, theorem IV.6.4.b]) provides us with a $p$-adic Lie group isomorphism

$$
\log : J_{1} \stackrel{\sim}{\longrightarrow} \mathfrak{p}^{g}
$$

which maps $J_{e}$ to $\left(\mathfrak{p}^{e}\right)^{g}$ for all $e\left(\right.$ where $\left(\mathfrak{p}^{e}\right)^{g}$ denotes the $g$-fold cartesian product of the set $\left.\mathfrak{p}^{e}\right)$. In particular, $J_{e} / J_{2 e} \simeq\left(\mathfrak{p}^{e} / \mathfrak{p}^{2 e}\right)^{g}$ is an Abelian group of exponent $p^{e}$.

Let now $x \in J\left(\mathbb{Z}_{q} / p^{e}\right)[m]$ be an $m$-torsion point known to accuracy $O\left(p^{e}\right)$. It has a unique $m$-torsion lift $\widetilde{x} \in J\left(\mathbb{Z}_{q} / p^{2 e}\right)[m]$, and its other lifts are of the form $\widetilde{x}^{\prime}=\widetilde{x}+y$ with $y \in J_{e} / J_{2 e}$. Therefore, if $N \in \mathbb{N}$ is such that $p^{e} \mid N$ and that $N \equiv 1 \bmod m$, then for any lift $\widetilde{x}^{\prime}$ of $x,[N] \widetilde{x}^{\prime}=\widetilde{x}$ is the $m$-torsion lift of $x$. This leads to the following algorithm:

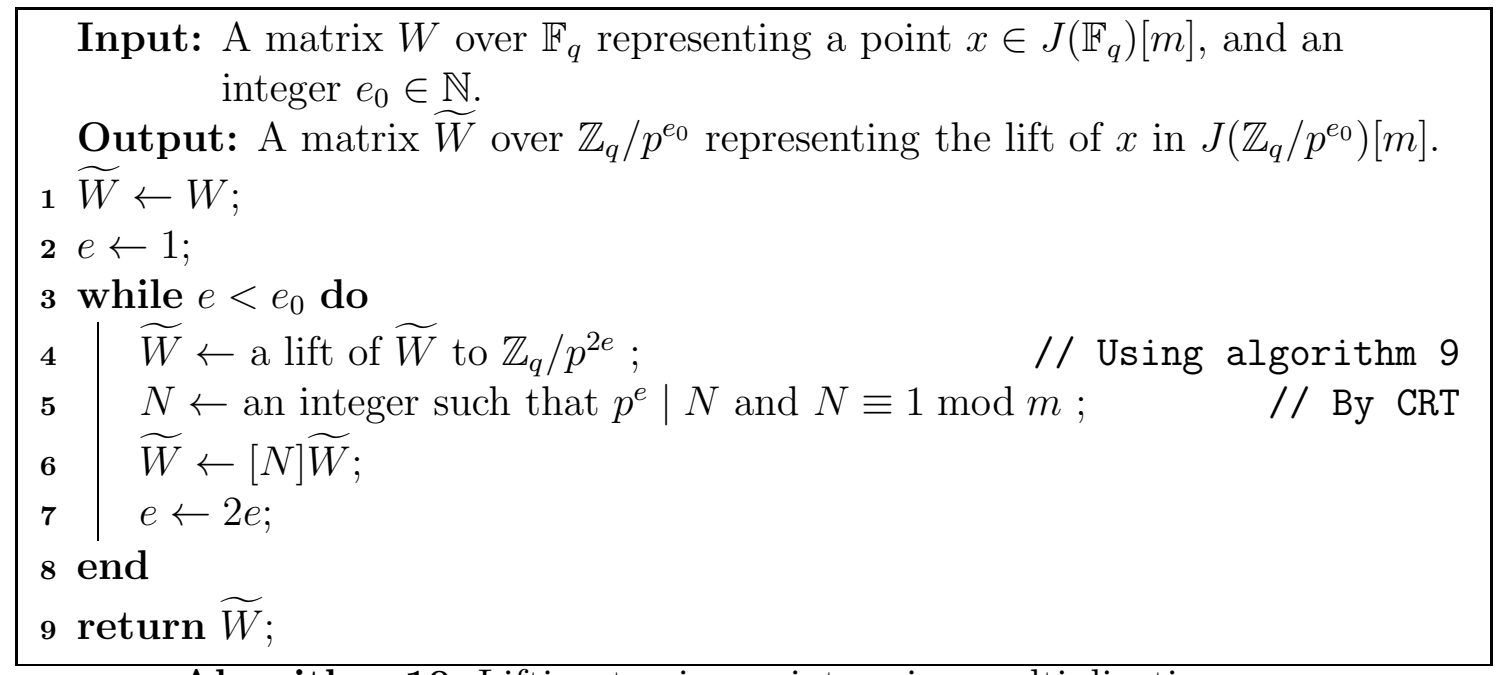

Algorithm 10: Lifting torsion points using multiplications 
The complexity of each iteration of the loop is $O\left(g^{\omega+3}+g^{\omega} \log \left(m p^{e_{0}}\right)\right)$ operations in $\mathbb{Z}_{q} / p^{O\left(e_{0}\right)}$, the terms coming respectively from algorithm 9 and from multiplication by $N=O\left(m p^{e_{0}}\right)$.

Remark 5.1. We could also apply algorithm 9 repeatedly so as to get a lift $\widetilde{W}$ of $W$ to precision $e_{0}$, and then multiply by $N$ where $p^{e_{0}-1} \mid N$ and $N \equiv 1 \bmod m$. However, this would be less efficient as we would perform all the multiplications at high accuracy. Anyway, it is better to use algorithm 11 for large $e$.

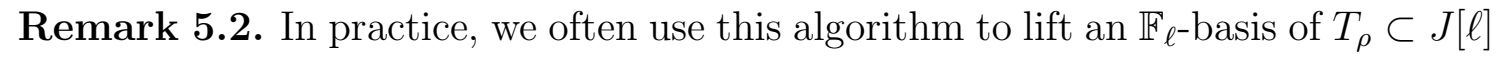
from $J\left(\mathbb{F}_{q}\right)[\ell]$ to $J\left(\mathbb{Z}_{q} / p^{e_{0}}\right)[\ell]$. In this case, we can afford to multiply each element of the basis by a nonzero scalar in $\mathbb{F}_{\ell}^{\times}$in the process, so we can save a bit of effort by suppressing the requirement that $N \equiv 1 \bmod \ell$ and simply taking $N=p^{e}$ at line 5 .

\subsection{Method 2: Using a coordinate chart at the origin}

We can use algorithm 7 as a coordinate chart $c^{\prime}: U \longrightarrow \mathbb{P}(V)$, where $U \subset J\left(\mathbb{Z}_{q}\right)$ is the preimage of a Zariski-dense subset of $J\left(\mathbb{F}_{q}\right)$. In the rest of this section, we assume that $U$ contains $0 \in J\left(\mathbb{Z}_{q}\right)$, since this is the case for "most" choices of divisors $E_{1}$ and $E_{2}$ used as parameters by algorithm 7 . Therefore, we are able to take a lift $\widetilde{W}$ of $W$ thanks to algorithm 9, multiply the corresponding point by $m$, and apply $c^{\prime}$ to see where in the vicinity of the origin of $J$ the result lies.

Ideally, we would like to compute the differential of the composition of the multiplication-by- $m$ map followed by $c^{\prime}$. Unfortunately this seems too complicated, so we settle for the following simpler approach: as explained in remark 4.5, a point $x \in J\left(\mathbb{Z}_{q} / p^{e}\right)$ possesses many different lifts $\widetilde{x} \in J\left(\mathbb{Z}_{q} / p^{e}\right)$ owing to the tangent space of $J$, so we can take sufficiently many such lifts $\widetilde{x}_{i}$, determine the coordinates of the $[m] \widetilde{x}_{i}$ thanks to $c^{\prime}$, and solve a linear system to deduce a "combination" $\widetilde{x}$ of the $\widetilde{x}_{i}$ such that $[m] \widetilde{x}=0$.

This idea translates into the following algorithm: 


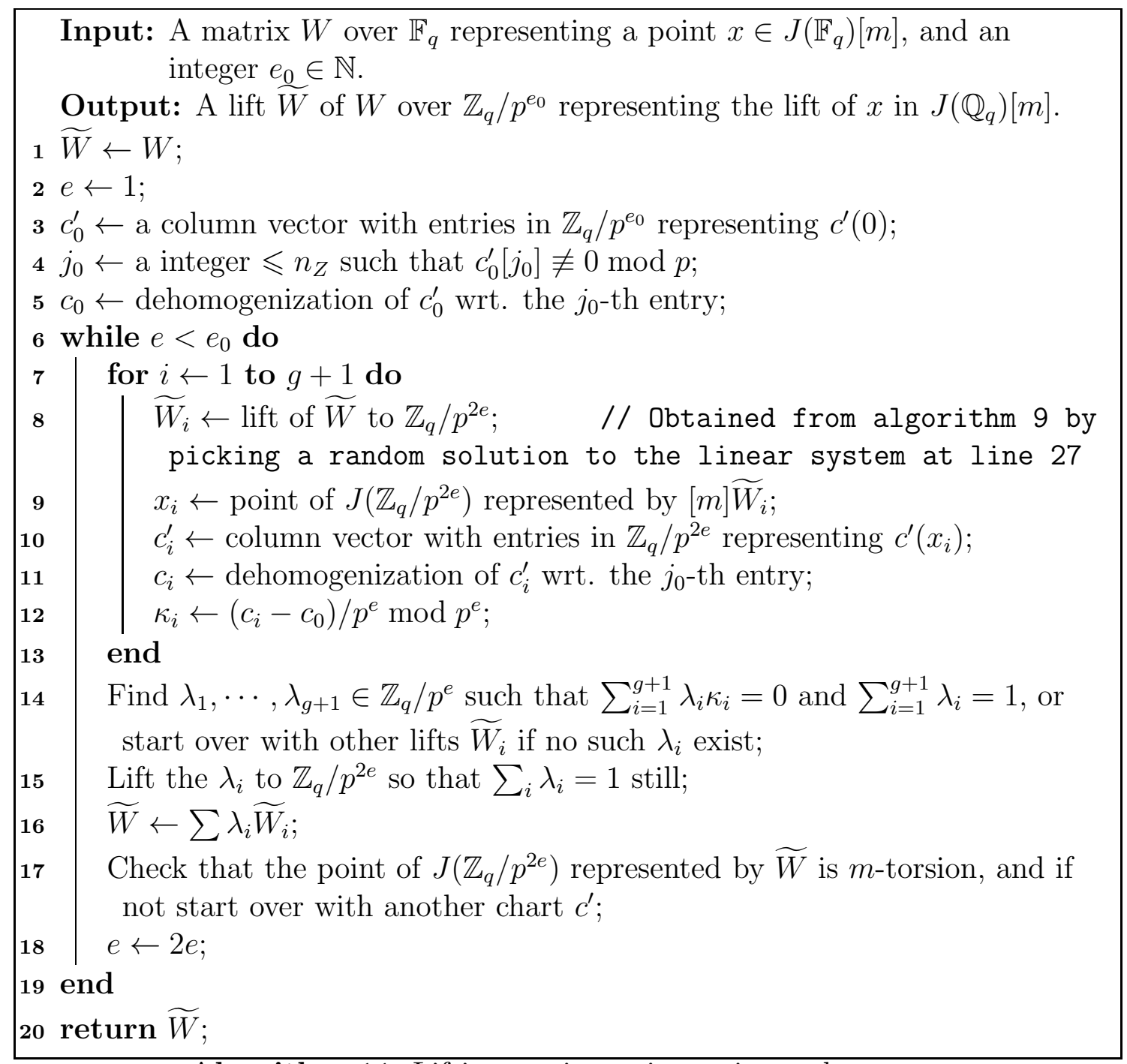

Algorithm 11: Lifting torsion points using a chart

Proof. Let us assume for now that the reduction mod $p$ of the differential of the "chart" $c^{\prime}: U \longrightarrow \mathbb{P}(V)$ at $0 \in J\left(\mathbb{Z}_{q}\right)$ has full rank $g$, which is extremely likely. Let $c: U \rightarrow \mathbb{Q}_{q}^{n_{Z}-1}$ denote $c^{\prime}$ followed by the embedding $\mathbb{P}(V) \hookrightarrow \mathbb{P}^{n_{Z}}$ induced by $V \hookrightarrow \mathbb{Q}_{q}^{n_{Z}}$ (cf. section 21) and then dehomogenized wrt. the $j_{0}$-th coordinate. Note that by our choice of $j_{0}, c$ is defined on the points of $J\left(\mathbb{Z}_{q}\right)$ that reduce to $0 \in J\left(\mathbb{F}_{q}\right)$, and actually sends them to $\mathbb{Z}_{q}^{n_{Z}-1}$. Thus for example at line 5 , we compute $c_{0}=$ $c(0) \bmod p^{e_{0}}$.

Suppose now that we have a matrix $W$ with coefficients in $\mathbb{Z}_{q} / p^{e}$ for some $e \in \mathbb{N}$ representing a point $x \in J\left(\mathbb{Z}_{q} / p^{e}\right)[m]$. Say that a lift of $W$ to $\mathbb{Z}_{q} / p^{2 e}$ is acceptable if it represents a point of $J\left(\mathbb{Z}_{q} / p^{2 e}\right)$, i.e. if it represents a subspace of $V$ and passes the membership test 4 (but it need not be $m$-torsion), and consider the map

$$
\begin{aligned}
\kappa:\{\text { Acceptable lifts of } W\} & \longrightarrow \frac{\left(\mathbb{Z}_{q} / p^{e}\right)^{n_{Z}-1}}{\widetilde{W}} \\
\widetilde{W}\left(x_{[m] \widetilde{W}}\right)-c_{0} & \longmapsto
\end{aligned}
$$

where $x_{[m] \widetilde{W}} \in J\left(\mathbb{Z}_{q} / p^{2 e}\right)$ denotes the point represented by the matrix $[m] \widetilde{W}$. Since $W$ represents an $m$-torsion point of $J\left(\mathbb{Z}_{q} / p^{e}\right), x_{[m] \widetilde{W}}$ reduces to $0 \in J\left(\mathbb{Z}_{q} / p^{e}\right)$ for all $\widetilde{W}$, so the expression $c\left(x_{[m] \widetilde{W}}\right)$ makes sense and is congruent to $c_{0} \bmod p^{e}$. Thus $\kappa$ is 
well-defined, and can be used to measure how a lift $\widetilde{W}$ of $W$ fails to be $m$-torsion, in that

The point of $J\left(\mathbb{Z}_{q} / p^{2 e}\right)$ represented by $\widetilde{W}$ is $m$-torsion $\Longleftrightarrow \kappa(\widetilde{W})=0 \bmod p^{e}$.

More precisely, let $\widetilde{W}$ be an (unknown) fixed acceptable lift of $W$ representing an $m$-torsion point of $J\left(\mathbb{Z}_{q} / p^{2 e}\right)$, so that $\kappa(\widetilde{W})=0 \bmod p^{2 e}$, and let $V_{0}$ be the matrix defined at line 6 of algorithm 9. This algorithm shows that any lift of $W$ of the form $\widetilde{W}+p^{e} V_{0} H$, where $H$ is matrix corresponding to a vector in the kernel of the linear system solved at line 27 of algorithm 9, is also an acceptable lift; and for such a lift, we have $\kappa\left(\widetilde{W}+p^{e} V_{0} H\right)=\kappa(\widetilde{W})+T\left(V_{0} H\right)=T\left(V_{0} H\right)$, where $T$ is the differential of $\kappa$, which is the reduction $\bmod p^{e}$ of a $\mathbb{Z}_{q}$-linear map of rank $g$ by our assumption on $c^{\prime}$.

Besides, the lifts constructed by algorithm 9 all have this form, so the $g+1$ lifts $\widetilde{W}_{i}$ considered at line 8 of algorithm 11 may be written as $\widetilde{W}_{i}=\widetilde{W}+p^{e} V_{0} H_{i}$. The $g+1$ vectors $\kappa_{i}=\kappa\left(\widetilde{W}_{i}\right)=T\left(V_{0} H_{i}\right)$ found at line 12 are necessarily linearly dependent, so at line 14 we can find scalars $\lambda_{i} \in \mathbb{Z}_{q} / p^{e}$ such that $\sum_{i=1}^{g+1} \lambda_{i} \kappa_{i}=0 \bmod p^{e}$ nontrivially. This relation can be rewritten as $\sum_{i=1}^{g} \lambda_{i}\left(\kappa_{i}-\kappa_{g+1}\right)+\left(\sum_{i=1}^{g+1} \lambda_{i}\right) \kappa_{g+1}=0$, so assuming that the $g$ vectors $\left(\kappa_{i}-\kappa_{g+1}\right)$ are linearly independent mod $p$ (which happens with high probability, else we start over), $\sum_{i=1}^{g+1} \lambda_{i}$ cannot be zero $\bmod p$; we can therefore rescale the $\lambda_{i}$ so that $\sum_{i=1}^{g+1} \lambda_{i}=1$. Define then

$$
\begin{aligned}
\widetilde{W^{\prime}} & =\sum_{i=1}^{g+1} \lambda_{i} \widetilde{W}_{i} \\
& =\widetilde{W}+\sum_{i=1}^{g+1} \lambda_{i}\left(\widetilde{W_{i}}-\widetilde{W}\right) \\
& =\widetilde{W}+p^{e} V_{0} \sum_{i=1}^{g+1} \lambda_{i} H_{i}
\end{aligned}
$$

as at line 16. This is an acceptable lift of $W$, which satisfies

$$
\kappa\left(\widetilde{W^{\prime}}\right)=T\left(V_{0} \sum_{i=1}^{g+1} \lambda_{i} H_{i}\right)=\sum_{i=1}^{g+1} \lambda_{i} \kappa_{i}=0 \bmod p^{e}
$$

it is therefore $m$-torsion by (5.3).

Now in all generality, as noted in remark 2.18, we cannot be completely certain that the reduction mod $p$ of the differential of $c^{\prime}$ at 0 has full rank $g$. If it is does not, then $c^{\prime}$ will not be a local immersion, so (5.3) is only a necessary but not sufficient condition. This nasty case does happen in practice, albeit very rarely, whence the test at line 17. In practice, this situation is revealed by the the fact that the vectors $\left(\lambda_{i}\right)_{i \leqslant g+1}$ satisfying $\sum_{i} \lambda_{i} \kappa_{i}=0$ at line 14 form a space of dimension more than 1 , so that it is sufficient to execute this test only at the first iteration of the while loop.

The complexity of each iteration of this loop is $O\left(g^{\omega+3}+g^{\omega+1} \log m\right)$ operations in $\mathbb{Z}_{q} / p^{O\left(e_{0}\right)}$.

Remark 5.4. One should not be surprised that the (a priori distinct) matrices $\widetilde{W}$ and $\widetilde{W^{\prime}}$ both represent the unique $m$-torsion lift of $x$ to $J\left(\mathbb{Z}_{q} / p^{2 e}\right)$, cf. remark 4.5. 
Remark 5.5. Of course, the computation of the $g+1$ lifts $\widetilde{W}_{i}$ at line 8 should not be done by calling algorithm $9 g+1$ times, but by modifying it so that it returns $g+1$ random acceptable lifts.

Remark 5.6. When lifting from $J\left(\mathbb{Z}_{q} / p^{e}\right)[m]$ to $J\left(\mathbb{Z}_{q} / p^{2 e}\right)[m]$, algorithm 11 performs $O\left(g^{\omega+3}+g^{\omega} \log \left(m p^{e}\right)\right)$ operations in $\mathbb{Z}_{q} / p^{O(e)}$, whereas algorithm 10 performs $O\left(g^{\omega+3}+g^{\omega+1} \log m\right)$. In order to lift a point from $J\left(\mathbb{F}_{q}\right)[m]$ to $J\left(\mathbb{Q}_{q}\right)[m]$, it is therefore reasonable to start with the method of algorithm 10 for low values of $e$, and to switch to the method of algorithm 11 when $e$ exceeds $g \log m / \log p$. If we use fast arithmetic, this results in a complexity of $\widetilde{O}(e a \log p) O\left(g^{\omega+3}+g^{\omega+1} \log m\right)$ bit operations to lift a point of $J\left(\mathbb{F}_{q}\right)[m]$ to $J\left(\mathbb{Z}_{q} / p^{e}\right)[m]$, where $q=p^{a}$. Note however that the loop at line 7 of algorithm 11 can very well be executed in parallel.

\section{Application to the computation of Galois rep- resentations}

We now finally come back to the initial problem, that of computing the Galois representation $\rho$ afforded by the subspace $T_{\rho} \subset J[\ell]$. Recall that we denote by $\Phi$ the Frobenius at $p$, and that we assume that we know the characteristic polynomial $\chi_{\rho}(x) \in \mathbb{F}_{\ell}[x]$ of $\rho(\Phi)$, as well as $L_{p}(x) \in \mathbb{Z}[x]$, that of $\Phi$ acting on $J$. Also recall that we assume that $\chi_{\rho}(x)$ and its cofactor $L_{p}(x) / \chi_{\rho}(x)$ are coprime in $\mathbb{F}_{\ell}[x]$.

\subsection{Splitting the representation}

The first thing to do is to determine $q=p^{a}$ so that the points of $T_{\rho}$ are defined over $J\left(\mathbb{Q}_{q}\right)$. The smallest such $a$ is of course the multiplicative order of $\rho(\Phi)$, which can be bounded thanks to the following result:

Proposition 6.1. Suppose $\chi_{\rho}(x)$ factors in $\mathbb{F}_{\ell}[x]$ as $\prod_{i} \chi_{i}(x)^{e_{i}}$, where the $\chi_{i}(x) \in$ $\mathbb{F}_{\ell}[x]$ are pairwise distinct irreducible polynomials of respective degrees $d_{i}$, and the $e_{i}$ are positive integers. Let $a_{i}$ be the multiplicative order of the class of $x$ in $\mathbb{F}_{\ell}[x] / \chi_{i}(x)$. Then the order of $\rho(\Phi)$ is of the form

$$
a=\ell^{u} \operatorname{lcm}\left(a_{i}\right)
$$

for some integer $u \leqslant \max _{i}\left\lceil\frac{\log e_{i}}{\log \ell}\right\rceil$, where $\lceil\cdot\rceil$ denotes rounding from above to the nearest integer.

Proof. Let $\phi=\rho(\Phi)$. Since the $\chi_{i}(x)^{e_{i}}$ are pairwise coprime, the $\mathbb{F}_{\ell}[\phi]$-module $\bar{T}_{\rho}$ decomposes as a direct sum $\bigoplus_{i} M_{i}$ of "generalised eigenspaces" $M_{i}=\operatorname{ker} \chi_{i}(\phi)^{e_{i}}$. The order of $\phi$ is thus the lcm of the order of the $\phi_{i}$, where $\phi_{i}=\phi_{\mid M_{i}}$. As $a_{i}$ divides $\ell^{d_{i}}-1$ for all $i$, the $a_{i}$ are coprime to $\ell$, so it is enough to show that for all $i$, the order of $\phi_{i}$ is of the form $\ell^{u_{i}} a_{i}$ for some integer $u_{i} \leqslant\left\lceil\frac{\log e_{i}}{\log \ell}\right.$.

The characteristic polynomial of $\phi_{i}$ is $\chi_{i}(x)^{e_{i}}$, so its eigenvalues in $\overline{\mathbb{F}_{\ell}}$ are the roots of $\chi_{i}(x)$, and are thus of multiplicative order $a_{i}$. Triangularising $\phi_{i}$ over $\overline{\mathbb{F}_{\ell}}$ thus shows that $\phi_{i}^{n}$ is unipotent iff. $a_{i}$ divides $n$. In particular, $a_{i}$ divides the order of $\phi_{i}$.

Write $\phi_{i}^{a_{i}}=1+N_{i}$ with $N_{i}$ nilpotent. The characteristic polynomial of $N_{i}$ is $x^{d_{i}}$, so $N^{d_{i}}=0$ by Cayley-Hamilton. Induction on $n$ reveals that $(1+N)^{\ell^{n}}=1+N^{\ell^{n}}$ for 
all $n \in \mathbb{N}$. So if $U_{i}=\left\lceil\frac{\log e_{i}}{\log \ell}\right\rceil$, so that $\ell^{U_{i}} \geqslant d_{i}$, then we have $\phi_{i}^{\ell^{U_{i}} a_{i}}=(1+N)^{\ell^{U_{i}}}=1$, which shows that the order of $\phi_{i}$ divides $\ell^{U_{i}} a_{i}$. Since this order is also divisible by $a_{i}$, the result follows.

Remark 6.2. It is clear that the bound $u \leqslant \max _{i}\left\lceil\frac{\log e_{i}}{\log \ell}\right\rceil$ is optimal.

In order to determine $a_{i}$, we simply have to find the primes $r$ dividing $n_{i}=$ $\ell^{d_{i}}-1$, and to test for each such $r$ whether $x^{n_{i} / r}$ is 1 in $\mathbb{F}_{\ell}[x] / \chi_{i}(x)$. Fortunately, in practice $\ell^{d_{i}}-1$ is not very large, so finding all these primes is not difficult.

In particular, in the case when $\chi_{\rho}(x)$ is squarefree, that is to say when $e_{i}=1$ for all $i$, then $u=0$, so we find that the order of $\rho(\Phi)$ is exactly $a=\operatorname{lcm}\left(a_{i}\right)$.

Remark 6.3. If $\chi_{\rho}(x)$ is not squarefree, then we obtain an upper bound on $a$ instead. The exact value of $u$, and thus of $a$, can then be determined by experimenting, but it is better to avoid this case by changing $p$ if possible.

In fact, if we know (or can afford to determine) $\chi_{\rho}(x)$ for not just one but several primes $p$, it is advisable for the efficiency of the rest of the computation to choose $p$ so that $a$ is minimal; cf. the examples in section 7 .

\subsection{Computing a basis of $T_{\rho} \bmod p$}

Now that we have chosen $q=p^{a}$, we can let $\overline{T_{\rho}} \subset J\left(\mathbb{F}_{q}\right)[\ell]$ be the reduction of $T_{\rho}$ $\bmod p$. The next step in the computation of $\rho$ consists in finding $\operatorname{dim} \overline{T_{\rho}}$ matrices $W$ over $\mathbb{F}_{q}$ representing an $\mathbb{F}_{\ell^{-}}$basis of $\overline{T_{\rho}}$. Since $\operatorname{dim} \overline{T_{\rho}}$ is known (for instance it is the degree of $\chi_{\rho}(x)$ ), our strategy consists in generating elements of $\overline{T_{\rho}}$ at random until we obtain a basis, which can be confirmed by algorithm 6 .

Let $N=\# J\left(\mathbb{F}_{q}\right)$, which can be computed explicitly as

$$
N=\operatorname{Res}\left(L_{p}(x), x^{a}-1\right)
$$

Let us factor

$$
N=\ell^{v} M
$$

where $M \in \mathbb{N}$ is coprime to $\ell$; we also define

$$
\psi(x)=L_{p}(x) / \chi_{\rho}(x) \in \mathbb{F}_{\ell}[x] .
$$

A simple-minded way to generate elements of $\overline{T_{\rho}}$ proceeds as follows:

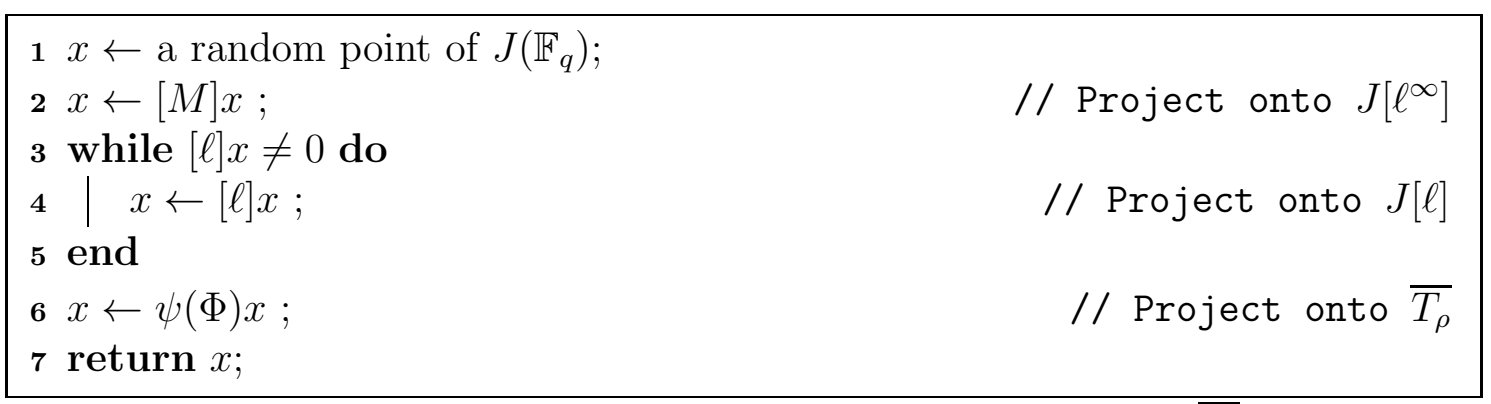

Algorithm 12: An unbalanced way to generate points of $\overline{T_{\rho}}$

Since $N=O\left(q^{g}\right)$ by the Weil bounds, the complexity of this algorithm is $O((g \log q+$ $a \log \ell) g^{\omega}$ ) operations in $\mathbb{F}_{q}$. 
Unfortunately, the points obtained this way are usually far from being equidistributed. For instance, suppose that $J\left(\mathbb{F}_{q}\right)\left[\ell^{\infty}\right]$ is of the form $\mathbb{Z} / \ell^{2} \mathbb{Z} \times \mathbb{Z} / \ell \mathbb{Z}$; then with high probability, after line $2, x$ has the form $(u, v)$ with $u$ of order $\ell^{2}$ and $v$ of order $\ell$, so that the loop at line 3 turns it into $(\ell u, 0)$, so we almost always get points of $J\left(\mathbb{F}_{q}\right)[\ell] \simeq \mathbb{Z} / \ell \mathbb{Z} \times \mathbb{Z} / \ell \mathbb{Z}$ whose second component is zero.

We can try to circumvent this issue by performing line 6 before the loop at line 3. but this does not help in case $\overline{T_{\rho}}$ itself has $\ell$-power torsion; besides, $\psi(x)$, which is computed mod $\ell$ only, will not be the correct cofactor, so the point we get in the end will not even lie in $\overline{T_{\rho}}$ in general!

In order to remedy this issue, [Bru13, section 3.9] suggests using the Kummer map

$$
\begin{aligned}
& J \longrightarrow J[\ell] \\
& x \longmapsto y^{\Phi}-y, \text { where }[\ell] y=x .
\end{aligned}
$$

Bruin shows that this process leads to uniform distribution in $J[\ell]$. However, this method requires enlarging the field $\mathbb{F}_{q}$, which considerably slows dow the computations, especially the multiplication-by- $M$ step (note that $M$ is of the order of magnitude of $q^{g}$ ).

We propose instead to modify algorithm 12 by including a process resembling Gaussian elimination. To demonstrate our idea, suppose again that $J\left(\mathbb{F}_{q}\right)\left[\ell^{\infty}\right] \simeq$ $\mathbb{Z} / \ell^{2} \mathbb{Z} \times \mathbb{Z} / \ell \mathbb{Z}$, and take $T_{\rho}=J[\ell]$ to simplify. If we start with two random points $x_{1}, x_{2} \in J$ and we apply algorithm 12, after line 2 these points become $x_{1}=$ $\left(u_{1}, v_{1}\right)$ and $x_{2}=\left(u_{2}, v_{2}\right) \in J\left[\ell^{\infty}\right]$, so unless one of the $u_{i}$ is $0 \bmod \ell$, line 3 leads us to $y_{1}=\left(\ell u_{1}, 0\right)$ and $y_{2}=\left(\ell u_{2}, 0\right)$, which fail to form a basis of $J[\ell]$. Nevertheless, we can turn this failure to our advantage! Indeed, thanks to algorithm 6 we can find $\lambda_{1}, \lambda_{2}$ not both 0 such that $\lambda_{1} y_{1}+\lambda_{2} y_{2}=0$, so dividing this relation by $\ell$ yields a new $\ell$-torsion point $x_{3}=\lambda_{1} x_{1}+\lambda_{2} x_{2}$, whose second component is nonzero with high probability.

Remark 6.4. Variants of this Gaussian elimination strategy appear in several places of the literature, such as Rav08].

This idea leads to the following algorithm, which performs very well in practice: 


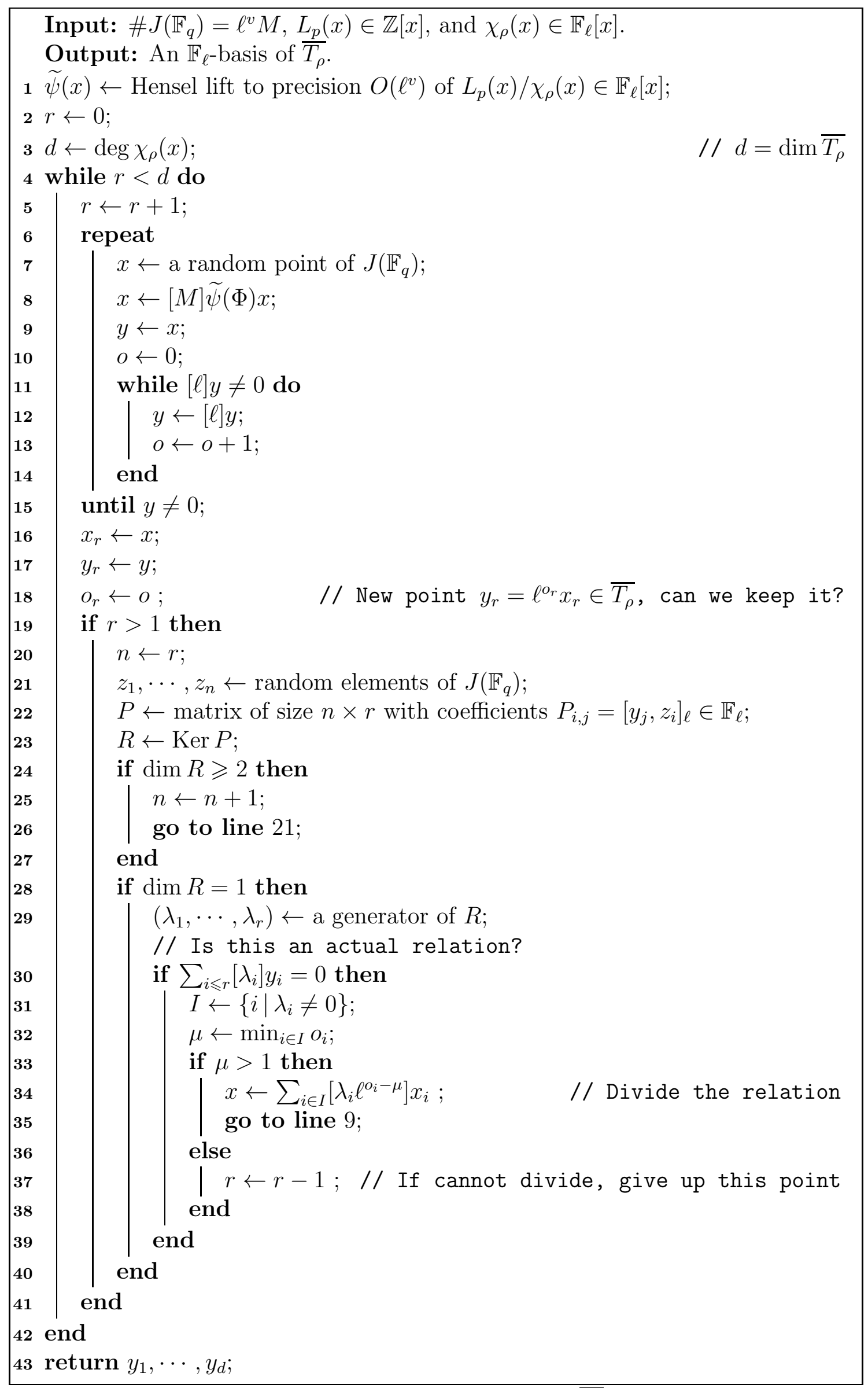

Algorithm 13: Finding a basis of $\overline{T_{\rho}}$ 
We now show the correctness of this algorithm and discuss its efficiency. Let us begin by introducing the main ideas.

6.2.1. First of all, we note that this algorithm needs to pick random points of $J\left(\mathbb{F}_{q}\right)$ at line 17, and also at line 21. Sophisticated methods to achieve this uniformly are presented in Bru13, but we content ourselves with a much cruder approach: we pick a random subset $S$ of $\left\{1, \cdots, n_{Z}\right\}$ of cardinality $d_{0}$, and compute by linear algebra the subspace $W$ of $V$ formed of vectors whose $i$-th coordinate vanishes for each $i \in S$. Indeed, this subspace represents the point $\left[\sum_{i \in S} P_{i}-D_{0}\right]$ of $J$, where the $P_{i}$ are as in section 2. Since these $P_{i}$ have been chosen at random, this approach performs very well in practice. In order to analyse the performance of algorithm 13, we will assume that the points thus obtained are uniformly distributed in $J\left(\mathbb{F}_{q}\right)$.

6.2.2. Let $L_{p}(x)=\widetilde{\chi}_{\rho}(x) \widetilde{\psi}(x)$ be the $\ell$-adic lift to accuracy $O\left(\ell^{v}\right)$ of the coprime factorisation $L_{p}(x) \equiv \chi_{\rho}(x) \psi(x) \bmod \ell$, and let $\Lambda=J\left(\mathbb{F}_{q}\right)\left[\ell^{v}\right] \leqslant J\left(\mathbb{F}_{q}\right)$ and $\Gamma=J\left(\mathbb{F}_{q}\right)\left[\ell^{v}, \widetilde{\chi}_{\rho}(\Phi)\right]=\Lambda\left[\widetilde{\chi}_{\rho}(\Phi)\right] \leqslant \Lambda$. In view of the Chinese remainder isomorphisms

$$
J\left(\mathbb{F}_{q}\right) \simeq J\left(\mathbb{F}_{q}\right)\left[\ell^{v}\right] \times J\left(\mathbb{F}_{q}\right)[M]=\Lambda \times J\left(\mathbb{F}_{q}\right)[M]
$$

and

$$
\Lambda \simeq \Lambda\left[\chi_{\rho}(\Phi)\right] \times \Lambda[\psi(\Phi)]=\Gamma \times \Lambda[\psi(\Phi)]
$$

if $g_{1}, \cdots, g_{r} \in J\left(\mathbb{F}_{q}\right)$ are uniformly chosen random points as in 6.2.1, then the points $h_{j}=[M] \widetilde{\psi}(\Phi) g_{j}$ are uniformly distributed in $\Gamma$.

6.2.3. Observe now that $\Gamma$ is a finite Abelian $\ell$-group, whose $\ell$-torsion subgroup $\Gamma[\ell]$ is precisely the space $\overline{T_{\rho}}$ that we are trying to generate. It is clear that sufficiently many elements $h_{i}$ of $\Gamma$ generated as in 6.2 .2 will form a generating set of $\Gamma$ with high probability; indeed, if $H=\left\langle h_{1}, \cdots, h_{k}\right\rangle$ is a strict subgroup of $\Gamma$, then it has index at least $\ell$, so $h_{k+1} \notin H$ with probability at least $1-\frac{1}{\ell}$.

6.2.4. Suppose thus that we have elements $h_{j} \in \Gamma$ which form a generating set. We can determine the order of each $h_{j}$ by repeatedly multiplying then by $\ell$ until the result is 0 . Let $n \in \mathbb{N}$ be such that the largest of these orders is $\ell^{n}$, so that the exponent of $\Gamma$ is $\ell^{n}$, and consider the projection morphism

$$
\pi=\left[\ell^{n-1}\right]: \Gamma \longrightarrow \Gamma[\ell]=\overline{T_{\rho}} .
$$

Let $P$ be the matrix such that $P_{i, j}=\left[\pi\left(h_{j}\right), z_{i}\right]_{\ell} \in \mathbb{F}_{\ell}$, where the $z_{i}$ are sufficiently many random elements of $J\left(\mathbb{F}_{q}\right)$ generated as in 6.2.1, and let $R$ be a matrix whose columns form an $\mathbb{F}_{\ell^{-}}$basis of Ker $P$. Then the columns of $R$ span a space containing all the $\mathbb{F}_{\ell^{-}}$-linear relations satisfied by the $\pi\left(h_{j}\right)$. Besides, for the same reason as in 6.2.3, the linear forms $y \mapsto\left[y, z_{i}\right]_{\ell}$ generate $\operatorname{Hom}_{\mathbb{F}_{\ell}}\left(\pi(\Gamma), \mathbb{F}_{\ell}\right)$ with high probability; we can check that this indeed the case by testing whether $\sum_{j} R_{j, k} \pi\left(h_{j}\right)=0$ for each $k$. If this is not the case, we generate more $z_{i}$, and try again; else the $h_{k}^{\prime}=$ $\sum_{j} \widetilde{R_{j, k}} h_{j}$ form a generating set of Ker $\pi=\Gamma\left[\ell^{n-1}\right]$, where $\widetilde{R_{j, k}}$ denotes an arbitrary lift to $\mathbb{Z}$ of $R_{j, k} \in \mathbb{F}_{\ell}$.

By iterating the process with $\Gamma\left[\ell^{n-1}\right]$ instead of $\Gamma$ and the $h_{k}^{\prime}$ instead of the $h_{j}$, we thus get generating sets for $\Gamma\left[\ell^{n-m}\right]$ for $m=1,2, \ldots$ until we get a generating set for $\Gamma\left[\ell^{1}\right]=\overline{T_{\rho}}$. 
6.2.5. Let now $h_{j} \in \Gamma$ be sufficiently many elements, so that they probably generate $\Gamma$ although we are not sure of that. By applying the method 6.2.4 to the $h_{j}$ (which amounts to working in the subgroup generated by the $h_{j}$ instead of $\Gamma$ ), either we find a generating set of $\overline{T_{\rho}}$, in which case we have reached our goal, so we stop; or we do not, in which case we conclude from 6.2.4 that our $h_{j}$ did not actually generate $\Gamma$, so we generate a few more of them as in 6.2 .2 and we try again.

These arguments show that this method quickly leads us to a generating set of $\overline{T_{\rho}}$ with high probability. Algorithm 13 is derived from these arguments, with a few extra optimizations. Namely, given one $h_{j} \in \Gamma$, instead of blindly computing $\pi\left(h_{j}\right)=$ $\left[\ell^{n-1} h_{j}\right]$, which is always $\ell$-torsion but could be 0 , we compute $\left[\ell^{o}\right] h_{j}$ where $o$ is the largest integer such that the result is non-zero; this provides us with more non-zero elements of $\overline{T_{\rho}}$, which help to obtain a generating set sooner. Besides, by introducing the $z_{i}$ one by one, and by throwing away the ones that yield a linear form which is in the span of the forms $\left[\cdot, z_{i}\right]_{\ell}$ corresponding to the previous $z_{i}$, we ensure that these forms remain linearly independent.

In detail, at line 7, we have obtained $r-1$ linearly independent points $y_{1}, \cdots, y_{r-1}$ of $\overline{T_{\rho}}$, with $y_{i}=\ell^{o_{i}} x_{i}$ and $x_{i} \in \Gamma$. After line [18, we have obtained a new nonzero point $y_{r}=\ell^{o_{r}} x_{r} \in \overline{T_{\rho}}$; however this point may be linearly dependent with $y_{1}, \cdots, y_{r-1}$, except of course if $r=1$. In order to determine whether this is the case, we examine these points through the linear forms $\left[\cdot, z_{i}\right]_{\ell}$ for $n$ random points $z_{i} \in J$, where initially $n=r$. The space $R$ we obtain contains the space $R^{\prime}$ of linear relations actually satisfied by $y_{1}, \cdots y_{r}$, but may be larger. Since $y_{1}, \cdots, y_{r-1}$ are linearly independent, $R^{\prime}$ has dimension at most 1 ; so if $\operatorname{dim} R \geqslant 2$ then $R \supsetneq R^{\prime}$, so we increase the number $n$ of linear forms and start our search for relations over. If $\operatorname{dim} R=1$, then either this candidate relation is a false positive, so the condition at line 30 is not satisfied, and $y_{1}, \cdots y_{r}$ are independent so can include $y_{r}$ in our partial basis of $\overline{T_{\rho}}$; or the candidate relation does hold, but then we can still try to get a new $\ell$-power torsion point by dividing the relation $R$ by a power of $\ell$. We take the result of this division as a new $x$ and start the whole process over, except if we cannot divide $R$ by $\ell$ because one of the $o_{i}$ is too small, in which case we give up the current $x$ and $y$ at line 37 and start over with a new random $x \in \Gamma$.

The complexity of this algorithm is $O\left((g \log q+(a+d) \log \ell) d g^{\omega}\right)$ where $d=\operatorname{dim} \rho$, assuming we have to give up the current $x$ a bounded number of times, and neglecting the cost of discrete logarithms in $\mu_{\ell}$ and of linear algebra over $\mathbb{F}_{\ell}$.

Remark 6.5. We can further improve the efficiency of the algorithm if, every time a new $y_{r}$ has been verified to be linearly independent of $y_{1}, \cdots, y_{r-1}$, we repeatedly apply the Frobenius $\Phi$ to $y_{r}$ and add the corresponding points to our partial basis of $\overline{T_{\rho}}$ as long as no linear dependence is detected. Indeed, the application of $\Phi$ by algorithm 8 is very fast. This idea is very similar to that presented in section 6.4 below.

Remark 6.6. In order to be able to use the "linearized" version of the Frey-Rück pairing, we need $\mathbb{F}_{q}$ to contain the $\ell$-th roots of unity (else this pairing would be trivial anyway). This is often the case, since the determinant of the representation $\rho$ usually involves the $\bmod \ell$ cyclotomic character due to the existence of the Weil pairing. In any case we can always extend $\mathbb{F}_{q}$, but this would considerably slow down all the computations. 


\subsection{The rest of the computation}

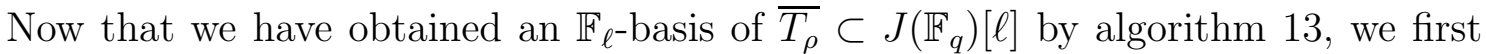
fix an $e \in \mathbb{N}$, and we lift our basis into a basis of $T_{\rho} \subset J\left(\mathbb{Q}_{q}\right)[\ell]$ to accuracy $O\left(p^{e}\right)$ by a combination of algorithms 10 and 11 as explained in remarks 5.6 and 5.2. We then use algorithm 3 to compute all the points of $T_{\rho}$, and we evaluate these points by algorithm 7, still at precision $O\left(p^{e}\right)$. All these computations can be massively parallelized, and their complexity is $\widetilde{O}(e a \log p) O\left(g^{\omega+3}+g^{\omega+1} \log \ell+g^{\omega} \ell^{d}\right)$ bit operations.

Finally, we form the monic polynomial $F(x)$ whose roots are the values in $\mathbb{Q}_{q}$ that we have just obtained. Since $T_{\rho}$ is globally invariant under Galois, $F(x)$ has coefficients in $\mathbb{Q}_{p}$, and these coefficients approximate rational numbers within $O\left(p^{e}\right)$. We can identify these rationals by rational reconstruction, provided that $e$ is large enough.

Remark 6.7. The value of $e$ is chosen from experience; see section 7 below for concrete examples. If $e$ is too low, rational reconstruction will most likely fail, but might also produce incorrect results. Therefore the output of our algorithm to compute $\rho$ is not guaranteed to be correct, and should be certified by methods such as Mas18a.

Arakelov theory could in principle provide us with bounds on the height of these rationals, but in practice these bounds are often extremely pessimistic, as noted in [Mas13].

\subsection{Saving effort thanks to the Frobenius}

We have already noted in remark 6.3 that for the efficiency of the computations, we should if possible choose the prime $p$ so that the degree $a=\left[\mathbb{F}_{q}: \mathbb{F}_{p}\right]$ is small. However, when this is not possible (e.g. because we cannot afford to compute the local factor $L_{p}(x)$ for more than a few small $p$ ), we can use the fact that $a$ is large to our advantage.

Indeed, $a$ is the order of Frobenius $\Phi \in \operatorname{Gal}\left(\mathbb{Q}_{q} / \mathbb{Q}_{p}\right)$. Since the evaluation algorithm 7 is (purposely!) Galois-equivariant, it commutes with $\Phi$. This means that when we want to apply it to all the points of $T_{\rho}$, we can apply it to only one point in each orbit of $\Phi$ of $T_{\rho}$, and recover the value of the other points simply by applying $\Phi$ to the result, which is instantaneous. In particular, we only need to compute one point of $T_{\rho}$ in each orbit instead of all of them, which saves an enormous amount of time, as the orbits of $\Phi$ on $T_{\rho}$ typically have length $a$.

Similarly, instead of lifting $p$-adically a whole $\mathbb{F}_{\ell^{-}}$basis of $T_{\rho}$ by the method presented in section 5 , we can lift a mere $\mathbb{F}_{\ell}[\Phi]$-generating set, after what we can use algorithm 8 to recover an $\mathbb{F}_{\ell^{-}}$-basis at high $p$-adic accuracy. The endomorphism induced by $\Phi$ on $T_{\rho}$ is often cyclic; when this is the case, we have to lift only one point instead of $\operatorname{dim} T_{\rho}$.

Remark 6.8. Although these ideas partly negate the inconvenience of working with a $p$ such that $a$ is large, choosing $p$ so as to minimize $a$ is still always a good idea. Indeed, the length of an orbit under $\Phi$ is at most $a$, so the idea presented above divides the amount of work by at most $a$, whereas the cost of computing in $\mathbb{F}_{q}$ and $\mathbb{Q}_{q}$ is super-linear in $a$. 


\section{Examples}

We have implemented the algorithms presented in this paper in the $\mathrm{C}$ language using the Pari/GP library, and we have applied them to a few examples. In most cases, the data we obtained could have been obtained in a much more direct way, but our goal here is to illustrate our methods.

The times given in this section are all CPU times (as opposed to wall times) on the author's laptop. The code is available on the author's personal web page [Mas] and as a Github repository Github.

\subsection{A modular representation with particularly little rami- fication}

Let $\rho: \operatorname{Gal}(\overline{\mathbb{Q}} / \mathbb{Q}) \longrightarrow \mathrm{GL}_{2}\left(\mathbb{F}_{7}\right)$ be either of the two mod 7 representations attached to the newform of weight 2 and level $\Gamma_{1}(13)$. In [Mas18b, section 6.3] we noted that these two representations are twists of each other, and that the corresponding projective representation $\pi: \operatorname{Gal}(\overline{\mathbb{Q}} / \mathbb{Q}) \longrightarrow \mathrm{PGL}_{2}\left(\mathbb{F}_{7}\right)$ corresponds to a particularly lightly ramified Galois number field of Galois group $\mathrm{PGL}_{2}\left(\mathbb{F}_{7}\right)$.

As a first demonstration of the methods presented in this article, we are going to compute $\rho$ and $\pi$. We thus take $\ell=7$ and $C$ the modular curve $C=X_{1}(13)$, whose Jacobian $J$ is such that $J[7]$ contains an $\mathbb{F}_{7}$-subspace $T_{\rho}$ of dimension 2 affording $\rho$. Note that although $J$ splits up to isogeny into the product of two copies of an elliptic curve defined over the real subfield of the 13th cyclotomic field, this decomposition does not occur over $\mathbb{Q}$, so computing $\rho$ through $J$ is reasonable.

The [LMFDB] informs us that $C$ is hyperelliptic of genus $g=2$ and admits the minimal model

$$
C: y^{2}+\left(x^{3}+x+1\right) y=x^{5}+x^{4} .
$$

We use this equation as input data; the fact that $C$ is hyperelliptic and is a modular curve is completely ignored by our algorithm.

The [LMFDB] also reveals that the only (up to Galois) newform of weight 2 and level 13 has coefficients in $\mathbb{Q}(\sqrt{-3})$, and provides us a few of these coefficients. By reducing these coefficients modulo one of the primes of $\mathbb{Q}(\sqrt{-3})$ above 7 , we get for each prime $p \notin\{7,13\}$ the characteristic polynomial of the image of the Frobenius at $p$ by $\rho$. Thanks to proposition 6.1, we can therefore look for a $p$ such that the points of $T_{\rho}$ are defined over $\mathbb{Q}_{p^{a}}$ for a small $a \in \mathbb{N}$.

We thus find that for $p=2$ we must take $a=8$ or 24 , depending on which of the primes above 7 we consider; for $p=3$ we need $a=48$ or 16 respectively, and so on. We spot that for $p=17$ we can take $a=6$ for either prime above 7 , so we choose to take $p=17$ and $a=6$; the respective characteristic polynomials being $x^{2}-2 x-1 \bmod 7$ and $x^{2}-x-2 \bmod 7$. Choosing for instance $\chi_{\rho}(x)=$ $x^{2}-2 x-1 \bmod 7$ (the other case being completely similar), we can now compute $\bmod p^{e}$ a polynomial $F(x)$ of degree $7^{2}-1$ defining $\rho$ for arbitrarily large $e \in \mathbb{N}$.

We take $e=32$. The initialization of Makdisi's algorithms to compute in $J$ at this accuracy takes about $50 \mathrm{~ms}$, the computation of an $\mathbb{F}_{7}$-basis of $T_{\rho} \bmod p$ takes $3.5 \mathrm{~s}$, lifting this basis to precision $O\left(17^{32}\right)$ takes $5.3 \mathrm{~s}$, computing $\mathbb{F}_{7}$-linear combinations representing all the orbits of $T_{\rho}$ under the Frobenius takes $1.8 \mathrm{~s}$, and evaluating a map $J \rightarrow \mathbb{A}_{1}$ at these points takes $4.9 \mathrm{~s}$. We then form the polynomial whose roots are these values, and manage to identify its coefficients as rationals 
(experiments show that this would have failed with $e=16$ instead of 32). All of this takes less than $10 \mathrm{~ms}$, and we obtain the polynomial

$$
\begin{aligned}
F(x)= & x^{48}-\frac{190}{11} x^{47}+\frac{11150}{121} x^{46}-\frac{70770}{121} x^{45}+\frac{192146}{121} x^{44}-\frac{865490}{121} x^{43}+15942 x^{42}-\frac{3378266}{121} x^{41}+\frac{7718056}{121} x^{40}-\frac{7242790}{121} x^{39} \\
& +\frac{161576962}{121} x^{38}-\frac{327843166}{121} x^{37}+\frac{1686874038}{121} x^{36}-\frac{2144483118}{121} x^{35}-\frac{3729825654}{121} x^{34}+\frac{18688301594}{121} x^{33} \\
& -\frac{23348747263}{121} x^{32}-\frac{183640041124}{121} x^{31}+\frac{334672016908}{121} x^{30}-\frac{1036146028660}{121} x^{29}+\frac{124260679100}{11} x^{28}+\frac{3580808205324}{121} x^{27} \\
& -\frac{32666349081700}{121} x^{26}+\frac{64676203524796}{121} x^{25}+\frac{94830437905560}{121} x^{24}-\frac{423358531106188}{121} x^{23}+\frac{261293629597764}{121} x^{22} \\
& +\frac{1780617009288708}{121} x^{21}-\frac{3884461192426292}{121} x^{20}+\frac{3993080217494308}{121} x^{19}-\frac{2118273836414700}{121} x^{18}+\frac{2868502387705524}{121} x^{17} \\
& +\frac{2992809907202955}{121} x^{16}-\frac{8017240457300370}{121} x^{15}+\frac{1032724415961478}{121} x^{14}+\frac{17530455675901702}{121} x^{13}+\frac{938376918522746}{121} x^{12} \\
& -\frac{21242403800528794}{121} x^{11}-\frac{6765741375874194}{121} x^{10}+\frac{812183325256186}{11} x^{9}+\frac{17495913080481536}{121} x^{8}-\frac{5797799442355694}{121} x^{7} \\
& -\frac{14434584737735526}{121} x^{6}+\frac{1659062818893114}{121} x^{5}+\frac{5000613835008606}{121} x^{4}-\frac{1428209615195030}{121} x^{3}-\frac{15369117321210}{11} x^{2} \\
& +\frac{76223729308434}{121} x+\frac{18369946454903}{77}
\end{aligned}
$$

which is irreducible over $\mathbb{Q}$ and defines a number field of discriminant $-7^{47} 13^{35}$. This is a strong hint that we have correctly identified the coefficients of $F(x)$, and this can be confirmed rigorously by the methods presented in [Mas18a].

By forming symmetric combinations (in this case, products) of the roots of $F(x)$ along vector lines in $T_{\rho}$, we get the polynomial

$$
G(x)=x^{8}+\frac{86}{11} x^{7}-\frac{35378}{11} x^{6}-28694 x^{5}+\frac{26301780}{11} x^{4}-\frac{729484894}{11} x^{3}+2233638278 x^{2}-\frac{410091777286}{11} x+\frac{18369946454903}{77}
$$

which is squarefree and therefore defines the projective representation $\pi$. Using $[$ Pari/GP]'s function polredabs to find a canonical polynomial whose root field is the same as that of $G(x)$ yields the polynomial

$$
G_{\text {red }}(x)=x^{8}-x^{7}+7 x^{6}+13 x-13
$$

whose Galois group is indeed $\mathrm{PGL}_{2}\left(\mathbb{F}_{7}\right)$. As predicted in [Mas18b, section 6.3], the root discriminant of the splitting field of this polynomial is $7^{7 / 8} 13^{5 / 6}=27.269 \ldots$, which is remarkably low. Besides, half of the coefficients of this polynomial are zero; we had encountered the same mysterious phenomenon in [Mas18b, section 5.1], and we still have no explanation.

Remark 7.1. Of course, since $C$ happens to be a modular curve, we could also have computed these representations by the techniques presented in Mas13. This would actually have taken longer, mainly because of the computation of the periods of the modular curve to high precision, and also because since these methods work over $\mathbb{C}$, we do not benefit much form the speedup introduced in section 6.4 as complex conjugation has order only 2 .

\subsection{The torsion of the Klein quartic}

Since it is overkill to use Makdisi's algorithms with hyperelliptic curves (where Mumford coordinates would be much more efficient), and in order to demonstrate the flexibility of our methods, we now experiment with the Klein quartic, given by the affine plane model

$$
C: x^{3} y+y^{3}+x=0 .
$$

This curve is isomorphic to the modular curve $X(7)$ over $\mathbb{Q}$, but again our algorithm completely ignores this fact. It has genus $g=3$, and according to [Elk99, section 2.3] 
its Jacobian $J$ is isogenous over $\overline{\mathbb{Q}}$ to the product of three copies of the elliptic curve $X_{0}(49)$, which has CM by $\mathbb{Z}\left[\frac{1+\sqrt{-7}}{2}\right]$. However, this decomposition does not occur over $\mathbb{Q}$, since not all local factors $L_{p}(x)$ of its $L$-function are perfect cubes. Besides, $C$ dominates $X_{0}(49)$, so the Galois representation afforded by the $\ell$-torsion of $X_{0}(49)$ also occurs in $J[\ell]$. Let us take $\ell=5$ for instance. By point counting, we spot that for $p=19$, the local factor $L_{p}(x)$ of $C$ factors as

$$
L_{19}(x)=\left(x^{2}+19\right)\left(x^{4}-19 x^{2}+19^{2}\right),
$$

so the Galois sub-module $T$ of $J[5]$ on which the Frobenius at 19 has characteristic polynomial $x^{2}+19$ must be isomorphic to the 5 -torsion of $X_{0}(49)$. Besides, luckily $x^{2}+19 \equiv x^{2}-1 \bmod 5$, so we can take $a=2$ only, i.e. the points of this sub-module are defined over $\mathbb{Q}_{19^{2}}$. Finding two points of $J\left(\mathbb{F}_{19^{2}}\right)$ forming an $\mathbb{F}_{5}$-basis of this sub-module takes $2.3 \mathrm{~s}$, lifting this basis to precision $O\left(19^{32}\right)$ takes $4.5 \mathrm{~s}$, forming $\mathbb{F}_{5}$-linear combinations representing all the orbits of $T$ under the Frobenius takes $1.2 \mathrm{~s}$, and evaluating the resulting points takes $3.4 \mathrm{~s}$. This 19 -adic accuracy is sufficient to identify the polynomial

$$
\begin{aligned}
F(x) & =x^{24}-\frac{307088989}{45285641} x^{23}+\frac{650200367}{45285641} x^{22}+\frac{871861629}{45285641} x^{21}-\frac{8986152651}{45285641} x^{20}+\frac{28369847905}{45285641} x^{19}-\frac{57494206833}{45285641} x^{18} \\
& +\frac{85829376881}{45285641} x^{17}-\frac{99861285810}{45285641} x^{16}+\frac{93389301219}{45285641} x^{15}-\frac{71688483976}{45285641} x^{14}+\frac{46005140069}{45285641} x^{13}-\frac{25177926620}{45285641} x^{12} \\
& +\frac{12024739657}{45285641} x^{11}-\frac{5124720540}{45285641} x^{10}+\frac{1973054346}{45285641} x^{9}-\frac{682322926}{45285641} x^{8}+\frac{207466033}{45285641} x^{7}-\frac{53995200}{45285641} x^{6}+\frac{11728577}{45285641} x^{5} \\
& -\frac{2073323}{45285641} x^{4}+\frac{288262}{45285641} x^{3}-\frac{29761}{45285641} x^{2}+\frac{2049}{45285641} x-\frac{361}{226428205}
\end{aligned}
$$

Thanks to $\mathrm{Pari} / \mathrm{GP}$, it is easy to check that this polynomial is irreducible over $\mathbb{Q}$ and defines the field of definition of a point of $X_{0}(49)[5]$. Of course, such a polynomial could have been obtained much more efficiently by working directly with the elliptic curve $X_{0}(49)$ instead of $C$ !

Given a prime $\ell$, we can also compute the representation

$$
\rho_{\ell}: \operatorname{Gal}(\overline{\mathbb{Q}} / \mathbb{Q}) \longrightarrow \operatorname{GSp}_{6}\left(\mathbb{F}_{\ell}\right)
$$

afforded by the whole of $J[\ell]$; however this yields polynomials of degree $\ell^{6}-1$, so we limit ourselves to $\ell \leqslant 3$.

Let us start with $\ell=2$. By point counting, we find that the local factor at $p=5$ is $L_{5}(x)=x^{6}+125$, which factors $\bmod 2$ as $(x+1)^{2}\left(x^{2}+x+1\right)^{2}$; therefore $J[2]$ is defined over $\mathbb{Q}_{5^{6}}$, so we take $p=5$ and $a=6$. Let $\Phi$ be the Frobenius at 5 . Getting an $\mathbb{F}_{2^{-}}$-basis of $J[2]$ over $\mathbb{F}_{5^{6}}$ and finding the matrix of $\Phi$ with respect to it takes $20 \mathrm{~s}$; the rational canonical form of this matrix is the cyclic permutation matrix

$$
\left(\begin{array}{llllll}
0 & 0 & 0 & 0 & 0 & 1 \\
1 & 0 & 0 & 0 & 0 & 0 \\
0 & 1 & 0 & 0 & 0 & 0 \\
0 & 0 & 1 & 0 & 0 & 0 \\
0 & 0 & 0 & 1 & 0 & 0 \\
0 & 0 & 0 & 0 & 1 & 0
\end{array}\right) \in M_{6}\left(\mathbb{F}_{2}\right)
$$

which shows that the $\Phi$-module $J[2]$ is isomorphic to $\mathbb{F}_{2}\left[C_{6}\right]$, and incidentally that the isogeny $J \sim X_{0}(49)^{3}$ cannot be defined over $\mathbb{Q}$. Since $J[2]$ is a cyclic $\mathbb{F}_{2}[\Phi]$-module, we can find a lift of this basis to precision $O\left(5^{64}\right)$ by lifting a single well-chosen point 
and taking the translates of the result under $\Phi$; this takes $8.4 \mathrm{~s}$. Forming $\mathbb{F}_{2}$-linear combinations representing all the $\Phi$-orbits of in $J[2] \backslash\{0\}$ takes $4 \mathrm{~s}$, and evaluating the resulting points takes $11.2 \mathrm{~s}$. It then takes less than $0.1 \mathrm{~s}$ to compute and identify the polynomial

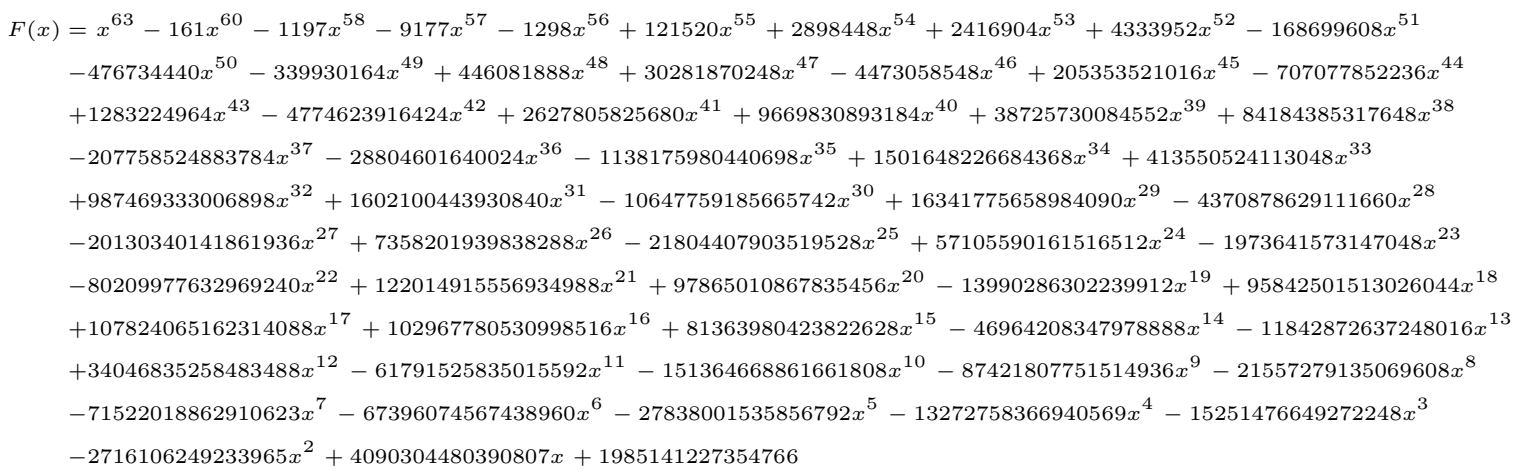

which is squarefree and factors over $\mathbb{Q}$ into one factor of degree 1 , one of degree 2 , two of degree 3 , and nine of degree 6.

It turns out that these factors all define a subfield of the 7 th cyclotomic field $\mathbb{Q}\left(\zeta_{7}\right)$, so, assuming that we have correctly identified the coefficients of $F(x)$, this means that the field of definition of $J[2]$ is $\mathbb{Q}\left(\zeta_{7}\right)$. This is an Abelian number field, and since 5 is a primitive root mod 7, its Galois group is generated by $\Phi$, the Frobenius at 5 . Therefore, the image of $\rho_{2}$ is cyclic of order 6 and generated by $\rho_{2}(\Phi)$, which is the matrix displayed above.

Finally, we redo the same computation, but with $\ell=3$. Finding a prime $p \notin\{3,7\}$ such that $J[3]$ is defined over a small extension of $\mathbb{Q}_{p}$ is not straightforward since the local factor $L_{p}(x)$ is always a cube mod 3 . In view of the fields in presence, we try $p=43$ since it is 1 both $\bmod 3$ and $\bmod 7$, and indeed a little experimentation shows that $J[3]$ is defined over $\mathbb{Q}_{43^{4}}$.

Getting an $\mathbb{F}_{3}$-basis of $J[3]$ over $\mathbb{F}_{43^{4}}$ takes $34 \mathrm{~s}$, lifting this basis to precision $O\left(43^{256}\right)$ takes 120 s, forming $\mathbb{F}_{3}$-linear combinations representing the orbits under the Frobenius takes $4 \mathrm{~m}$, and evaluating the resulting points takes $9 \mathrm{~m} 20 \mathrm{~s}$. We obtain a polynomial $F(x)$ of degree 728 whose coefficients are rationals with numerators of up to 163 digits and always the same 133-digit denominator up to small primes, and which is squarefree and factors over $\mathbb{Q}$ into one factor of degree 8 , four of degree 24 , and thirteen of degree 48.

These factors all define subfields of a number field $L$ of degree 48 which is Galois, totally imaginary, and has discriminant $3^{42} 7^{44}$ (in particular its root discriminant is $3^{7 / 8} 7^{11 / 12}=15.565 \cdots$, which is very close to the record given in table 3 p. 134 of Odl90]).

Thanks to Magma and GAP18, we can determine that its Galois group is a non-Abelian nilpotent group of order 48, isomorphic to the direct product of a cyclic group of order 3 and of the normalizer of a non-split Cartan of $\mathrm{GL}_{2}\left(\mathbb{F}_{3}\right)$. This is not surprising, since the image of the mod 3 representation attached to the elliptic curve $X_{0}(49)$ is precisely this normalizer. In fact, $L$ turns out to be the compositum of the real subfield $\mathbb{Q}\left(\zeta_{7}\right)^{+}$of the 7-th cyclotomic field and of the field of definition of the 3-torsion of $X_{0}(49)$. Besides, the factor of degree 8 of $F(x)$ defines the field of definition of a point of $X_{0}(49)$ [3], and the factors of degree 24 define the compositum of this field and of $\mathbb{Q}\left(\zeta_{7}\right)^{+}$. 


\subsection{A higher genus example}

Finally, in Mas19], we used the methods presented in this article to compute a $\mathrm{GL}_{3}\left(\mathbb{F}_{9}\right)$-valued Galois representation afforded by a 6-dimensional $\mathbb{F}_{3}$-subspace of the 3 -torsion of the Jacobian of a curve of genus $g=7$. Unlike the previous computations that took place on the author's laptop, this computation took place on a computing cluster with 32 cores provided by Warwick mathematics institute. It only took about one hour of wall time thanks to parallelisation, which demonstrates that our methods are also suitable to higher genera. The CPU times were as follows: getting a basis of this 6-dimensional subspace of $J\left(\mathbb{F}_{q}\right)[3]$ (with $q=11^{14}$ in this case) took about $2 \mathrm{~h}$, lifting this basis to $p$-adic accuracy $O\left(11^{1024}\right)$ took $3.5 \mathrm{~h}$, forming linear combinations of these points representing all the 104 orbits under the Frobenius at $p=11$ took $6 \mathrm{~h}$, and evaluating these points took $12.5 \mathrm{~h}$.

It is interesting to note that even for such a large genus, the step of highest complexity, namely lifting $p$-adically the torsion points by a combination of algorithms 9, 10, and 11 whose complexity is $O\left(g^{\omega+3}\right)$, is actually less time-consuming than the subsequent steps, even though their complexity is only $O\left(g^{\omega}\right)$. This is not so surprising, since the $O\left(g^{\omega+3}\right)$ complexity is only due to the very last lines of algorithm 9 .

In view of these encouraging results, we plan to use this new $p$-adic method to try to beat the genus records set in Mas13] for Galois representations attached to modular forms in the near future. 


\section{References}

[AGS18] Abelard, Simon; Gaudry, Pierrick; Spaenlehauer, Pierre-Jean, Improved Complexity Bounds for Counting Points on Hyperelliptic Curves. Foundations of Computational Mathematics, Springer Verlag, DOI : 10.1007/s10208-018-9392-1.

[BBB94] Bergeron, F.; Berstel, J.; Brlek, S., Efficient computation of addition chains. J. Théor. Nombres Bordeaux 6 (1994), no. 1, 21-38.

[Bru13] Bruin, Peter, Computing in Picard groups of projective curves over finite fields. Mathematics of Computation 82 (2013), 1711-1756.

[CF+05] Cohen, Henri; Frey, Gerhard; Avanzi, Roberto; Doche, Christophe; Lange, Tanja; Nguyen, Kim; Vercauteren, Frederik, eds., Handbook of elliptic and hyperelliptic curve cryptography. CRC press, 2005

[Dok13] Dokchitser, Tim and Vladimir, Identifying Frobenius elements in Galois groups. Algebra \& Number Theory, Volume 7, Number 6 (2013), $1325-1352$.

[Elk99] Elkies, Noam D., The Klein quartic in number theory. The eightfold way, 1999, vol. 35, 51-101.

[FR94] Frey, Gerhard; Rck, Hans-Georg, A remark concerning m-divisibility and the discrete logarithm in the divisor class group of curves. Mathematics of Computation 62 (1994), 865-874.

[GAP18] The GAP Group, GAP - Groups, Algorithms, and Programming, Version $4.9 .2 ;$ 2018. (https://www.gap-system.org)

[Github] Mascot, Nicolas, Github repository https://github.com/nmascot/LiftTors.

[Hes02] Hess, Florian, Computing RiemannRoch spaces in algebraic function fields and related topics. Journal of Symbolic Computation 33, no. 4 (2002), 425-445.

[How86] Howell, John A., Spans in the module $\left(\mathbb{Z}_{m}\right)^{s}$. Linear and Multilinear Algebra, 19:1, 67-77, 1986. DOI: 10.1080/03081088608817705

[KM04] Khuri-Makdisi, Kamal, Linear algebra algorithms for divisors on an algebraic curve. Math. Comp. 73 (2004), no. 245, 333-357.

[KM07] Khuri-Makdisi, Kamal, Asymptotically fast group operations on Jacobians of general curves. Mathematics of Computation 76 (2007), no. 260, 2213-2239.

[LMFDB] The LMFDB Collaboration, The L-functions and Modular Forms Database. http://www.lmfdb.org

[Magma] Bosma, Wieb; Cannon, John; Playoust, Catherine, The Magma algebra system. I. The user language J. Symbolic Comput., 24 (1997), 235265. 
[Mas] Mascot, Nicolas, Personal web page. https://staff.aub.edu.1b/ nm116/.

[Mas13] Mascot, Nicolas, Computing modular Galois representations. Rendiconti del Circolo Matematico di Palermo, Volume 62, Number 3, 451-476.

[Mas18a] Mascot, Nicolas, Certification of modular Galois representations. Mathematics of Computation 87 (2018), 381-423

[Mas18b] Mascot, Nicolas, Companion forms and explicit computation of $\mathbf{P G L}_{2}$ number fields with very little ramification. Journal of Algebra, Volume 509, 1 September 2018, 476-506

[Mas19] Mascot, Nicolas, Explicit computation of a Galois representation attached to an eigenform over $\mathrm{SL}_{3}$ from the $\mathrm{H}^{2}$ étale of a surface. ArXiv preprint 1810.05885, 2019.

[Mil04] Miller, Victor S., The Weil Pairing, and Its Efficient Calculation Journal of Cryptology, September 2004, Volume 17, Issue 4, 235-261

[Od190] Odlyzko, Andrew M., Bounds for discriminants and related estimates for class numbers, regulators and zeros of zeta functions: a survey of recent results. Sém. Théor. Nombres Bordeaux (2), 1990, vol. 2, no 1, 119-141.

[Pari/GP] The PARI Group, PARI/GP development version 2.10.0, Bordeaux, 2018, http://pari.math.u-bordeaux.fr/

[Rav08] Ravnshøj, Christian Robenhagen, Generators of Jacobians of Genus Two Curves. IACR Cryptology ePrint Archive 2008: 70, https://eprint.iacr.org/2007/150.pdf.

[Sil09] Silverman, Joseph H., The arithmetic of elliptic curves, second edition. Graduate Texts in Mathematics, 106. Springer- Verlag, New York, 2009.

[SM98] Storjohann, Arne; Mulders, Thom, Fast algorithms for linear algebra modulo $N$. European Symposium on Algorithms, 139-150. Springer, Berlin, Heidelberg, 1998. 